\title{
Geometry-dependent conductance and noise behavior of a graphene ribbon with a series of randomly spaced potential barriers
}

\author{
Paolo Marconcini ${ }^{1, \text { a) }}$ and Massimo Macucci ${ }^{1, b}$ ) \\ ${ }^{1}$ Dipartimento di Ingegneria dell'Informazione, Università di Pisa, \\ Via Girolamo Caruso 16, 56122 Pisa, Italy
}

(Dated: 14 May 2019)

\begin{abstract}
We perform an envelope-function based numerical analysis of the effect of a sequence of randomly spaced potential barriers on the conductance and shot noise of an armchair graphene ribbon. The behavior is dominated by Klein tunneling and by resonant tunneling, and strongly depends on the geometrical details of the device. Klein tunneling effectively filters the modes which can propagate through the device. For a large number of cascaded barriers, this gives rise to different transport regimes for metallic and semiconducting ribbons, with diverging shot noise behaviors. Resonant tunneling is instead energy selective and has quite a different effect depending on whether the barriers are identical or not. We explore also the effect of tilting the barriers with respect to the ribbon edges, observing a transition towards a diffusive transport regime and a one-third shot noise suppression. We investigate this effect and we find that it takes place also in more traditional semiconducting materials. The results of our analysis could be instrumental for the fabrication of mode-filtering and energy-filtering graphene-based nanodevices. Moreover, our study highlights the importance of the measurement of shot noise as a probe for the nature of the transport regime.
\end{abstract}

\section{INTRODUCTION}

Starting from the seminal work of Geim and Novoselov ${ }^{1,2}$, many efforts have recently focused on the study of graphene. This two-dimensional material has many fascinating properties, including high electrical and thermal conductance, mechanical strength and flexibility, transparency and chemical stability, which make it interesting in different application fields, spanning from the fabrication of composites, coatings and membranes, to energy storage systems, sensors, electronic, optoelectronic, and phonon-based devices ${ }^{3-13}$. Graphene is made up of an hexagonal lattice of $s p^{2}$ hybridized carbon atoms. The unit cell of this lattice contains two inequivalent atoms, generally specified with the letters $A$ and $B$, which give rise to two triangular sublattices ${ }^{14-16}$. Unconfined monolayer graphene has no energy gap: its highest valence band and lowest conduction band touch each other at two inequivalent degeneration points $\vec{K}$ and $\vec{K}^{\prime}$ (Dirac points) of the reciprocal space. Around these points, the dispersion relations are linear (which means that graphene has a zero effective mass) and the behavior of graphene can be approximately described in terms of four envelope functions $F_{\beta}^{\vec{\alpha}}$ (which correspond to the two sublattices $\beta=A, B$ and to the two Dirac points $\left.\vec{\alpha}=\vec{K}, \vec{K}^{\prime}\right)^{14}$. Due to the particular lattice structure of graphene, these functions $F_{\beta}^{\vec{\alpha}}$ have to satisfy an envelope-function equation which formally coincides with the Dirac-Weyl equation ${ }^{17,18}$, which also describes the behavior of massless spin-1/2 particles in relativistic quantum mechanics. As a consequence, graphene exhibits very peculiar properties, previously expected only at relativistic speeds. Among these exotic phenomena are ${ }^{19,20}$ Klein tunneling ${ }^{21-26}$ (the property that a particle impinging against an orthogonal barrier has a unitary probability to transmit across it, what-

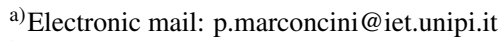

b)Electronic mail: m.macucci@mercurio.iet.unipi.it
}

ever its width and height), Zitterbewegung ${ }^{27}$ (an oscillatory behavior deriving from the interference between the components of the wave packet with positive and negative energy), the Anomalous Integer Quantum Hall Effect ${ }^{2,28-30}$ (an unusual type of Quantum Hall effect characterized, among other peculiarities, by the presence of a zero-energy Landau level), and Veselago lensing ${ }^{31,32}$ (an optical phenomenon based on negative refraction).

In graphene, the conductance through a single tunnel barrier is dominated by Klein tunneling and by resonant tunneling through the states which are quasi-localized inside the barrier $^{21,33-38}$.

In this paper we will focus on ribbons with armchair edges, which can present either a (quasi-)metallic or a semiconducting behavior, depending on the exact number of dimer lines across their width ${ }^{14,39,40}$ (while zigzag ribbons are always (quasi-)metallic; for a discussion on the effect of a potential step or barrier in a zigzag ribbon see Refs. ${ }^{41-44}$ ).

In particular, we will numerically simulate the transport behavior of an armchair graphene ribbon with a series of randomly spaced potential barriers. This potential profile could be obtained, for example, by negatively biasing a series of parallel top gates (separated from the underlying ribbon by a dielectric layer) with random inter-gate distances (see the sketch in Fig. 1). We will analyze the role that Klein tunneling and resonant tunneling play in this structure and the dependence on the specific geometrical details. Moreover, we will study how this affects the shot noise properties of the device.

As we will show, the structure has different properties from the case of evenly spaced tunnel barriers (the conductance behavior of which has been analyzed in Refs. $\left.{ }^{45-54}\right)$. Indeed, the presence, in the structures we will consider, of different random inter-barrier distances excludes the presence of oscillations due to the resonance with the states quasi-localized in the regions between adjacent barriers and to the appearance of new Dirac points, oscillations which instead have been predicted and observed in the case of evenly spaced tunnel barriers ${ }^{45,49-52}$. 


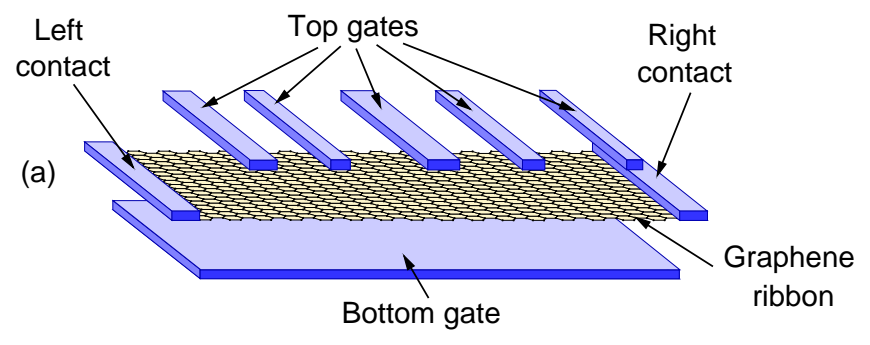

(b)

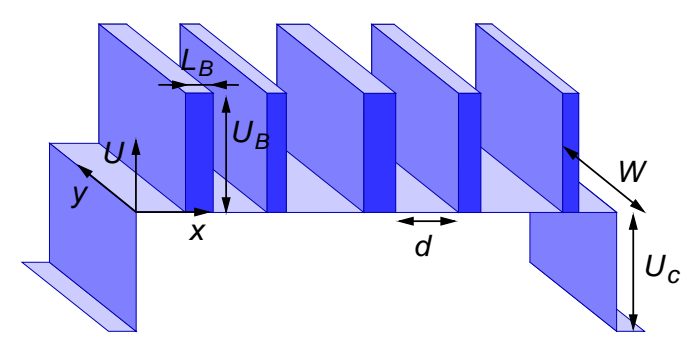

FIG. 1. Armchair graphene ribbon with a possible gate configuration (a) leading to the potential profile (b) that we are analyzing.

In our devices, Klein tunneling will operate a selection of the modes propagating through the ribbon, on the basis of their angle of incidence. For a sufficiently large number of barriers the behavior will differ depending whether the ribbon is semiconducting or metallic. In a semiconducting ribbon, no transport mode has a wave vector (measured with respect to the Dirac points) with zero transverse component, and thus travels in a direction exactly orthogonal to the barriers. Therefore, we will reach a localized transport regime, with zero conductance and no suppression of the shot noise power spectral density (with respect to the value $2 e I$ predicted by Schottky ${ }^{55}$ in the case of charge carriers propagating independently through the device, where $e$ is the elementary charge and $I$ is the average current value). In a metallic ribbon, instead, one transport mode is exactly orthogonal to the barriers and thus is able to ballistically cross all the barriers with unit transmission. Therefore, the normalized conductance $G$ will approach the quantum of conductance $G_{0}=2 e^{2} / h$ (where $h$ is Planck's constant) and shot noise will vanish.

On the other hand, resonant tunneling through the states quasi-localized inside each barrier will operate a selection of the charge carriers on the basis on their energy. The modes with an energy equal to that of the quasi-bound states inside the barrier will experience enhanced transmission, while the others will be suppressed. If the potential profile is made up of identical barriers, each barrier will perform the same selection and the overall device will preserve such a dependence of the transmission on the energy. Instead, in the case of different barriers a more uniform behavior as a function of energy will appear.

We will finally consider the case of barriers which are tilted with respect to the ribbon edges, although all by the same angle (only the analysis of the transport behavior of a single tilted barrier has been previously reported in the literature ${ }^{56}$ ). In this case, the barriers, introducing mode-mixing in the device, make it possible to reach the diffusive transport regime. In particular, this gives rise to a shot noise suppression factor
$F$ (Fano factor) equal to $1 / 3$, where the Fano factor is defined as the ratio of the actual value of the shot noise power spectral density $S_{I}$ to the "full" value $2 e I$ predicted by Schottky. We have verified that this conclusion is valid also in devices made up of more traditional semiconducting materials, for example in quantum wires fabricated by confining the two-dimensional electron gas (2DEG) of GaAs/AlGaAs heterostructures. This behavior differs from what we reported in the past for 2DEGbased devices ${ }^{57}$, for barriers orthogonal to the transport direction. Indeed, in that case the absence of mode-mixing made it actually impossible to reach the diffusive regime, and thus increasing the number of barriers a direct crossover from the ballistic to the localized regime was observed ${ }^{57,58}$.

\section{SIMULATION METHOD}

We have performed our analysis using our in-house developed envelope-function based transport simulator ${ }^{59}$, which can compute conductance and noise in graphene structures with different geometries and potential profiles ${ }^{38,60,61}$. As we have previously stated, around the Dirac points the wave function of graphene can be written in terms of four envelope functions, which have to satisfy the Dirac-Weyl equation. In order to simplify the calculations, the code divides the armchair graphene ribbon into a series of sections, where the Dirac-Weyl equation can be more easily solved. Each section has a width $W$ (along the transverse direction $y$ ) equal to that of the ribbon, while its length in the transport direction $x$ is chosen in such a way as to be able to consider the potential $U$ in each section approximately independent of $x$. With this choice, the envelope functions inside each section can be written as the product of a transverse component $\Phi_{\beta}^{\vec{\alpha}}(y)$ and of a plane wave propagating in the transport direction $e^{i \kappa_{x} x}$ : $F_{\beta}^{\vec{\alpha}}(x, y)=e^{i \kappa_{x} x} \Phi_{\beta}^{\vec{\alpha}}(y)$. Substituting these expressions into the Dirac-Weyl equation, with Dirichlet boundary conditions for the graphene wave function at the ribbon edges, we obtain a differential problem in the transverse components $\Phi_{\beta}^{\vec{\alpha}}(y)$ and in the longitudinal wave vector $\kappa_{x}$. Then, this differential problem can be transformed into an equivalent one with periodic boundary conditions ${ }^{59,62,63}$. This makes it possible to solve the problem in the reciprocal space, overcoming the issue of fermion doubling and increasing the efficiency of the numerical procedure ${ }^{59}$. In particular, everything is reduced to an eigenproblem where the eigenvectors are made up of the Fourier components of a quantity related to the transverse components of the envelope functions, while the eigenvalues are the longitudinal wave vectors. Within each of the sections, we solve this problem, obtaining the modes which propagate in that part of the device. Then, we compose the results obtained in the different sections using a recursive scattering matrix approach. At the interface between adjacent sections, we enforce the continuity of the wave functions on both the $A$ and $B$ sublattices. In particular, exploiting the linearity of the problem, we imagine to inject a single mode at a time from the left and from the right side of the interface, we enforce the continuity across the interface and we project the resulting 
relations onto a basis of sine functions. In this way, we obtain a system of linear equations in the transmission and reflection coefficients across the interface. These coefficients represent the elements of the scattering matrix of the region surrounding the interface. The scattering matrices of the different regions of the device are then combined, using standard techniques ${ }^{64}$, in such a way as to obtain the scattering matrix of the overall device and in particular its transmission matrix $t$. From the transmission matrix, the value of the conductance $G$ and of the Fano factor $F$ can be obtained using the Landauer-Büttiker formulas ${ }^{65-67}$ :

$$
G=\frac{2 e^{2}}{h} \sum_{i} w_{i}, \quad F=\frac{S_{I}}{2 e I}=\frac{\sum_{i} w_{i}\left(1-w_{i}\right)}{\sum_{i} w_{i}},
$$

where $w_{i}$ is the generic eigenvalue of the matrix $t^{\dagger} t$ and the sums run over all the modes propagating in the input and output leads.

The results are uniformly averaged over the energy range delimited by the electrochemical potentials of the input and output leads (assuming to operate at low temperature, we consider a steplike behavior of the Fermi-Dirac distribution function). In the case of the Fano factor, the averages have to be performed separately for the numerator and for the denominator of the expression of $F$ in Eq. (1) (and thus for $S_{I}$ and $I$ ), consistently with the experimental procedure for its measurement ${ }^{68}$.

In our calculations, we have included the effect of the contacts considering regions with a negative potential energy $U_{c}$ (in our simulations equal to $-0.2 \mathrm{eV}$ ) at the entrance and exit of the device. However, the introduction of the two regions with negative potential energies has a significant effect on the results only in a small energy interval around zero (the interval in which otherwise no mode would be injected into the device).

For each simulation, the length of the ribbon has been chosen in such a way as to accommodate the investigated number of barriers, leaving a distance between the contacts and the first/last barrier of the order of the average interbarrier distance.

The simulation code is written in Fortran and exploits Lapack routines ${ }^{69}$. The simulations have been performed on a 100 -core cluster based on Intel Xeon processors, parallelizing the calculations performed for different energies.

\section{NUMERICAL RESULTS}

Unless otherwise specified, in our calculation we have considered graphene ribbons with a width of $200 \mathrm{~nm}$, with armchair edges and with a potential profile given by a series of $0.3 \mathrm{eV}$ high tunnel barriers (even though we have verified that the results are valid also for smaller ribbons). We have assumed an energy difference of $0.4 \mathrm{meV}$ between the Fermi levels of the input and output leads, much greater than $k_{B} T$ (with $k_{B}$ the Boltzmann constant and $T$ the absolute temperature, assumed less than $1 \mathrm{~K}$ ). However, further simulations performed at higher temperature have shown that the selective
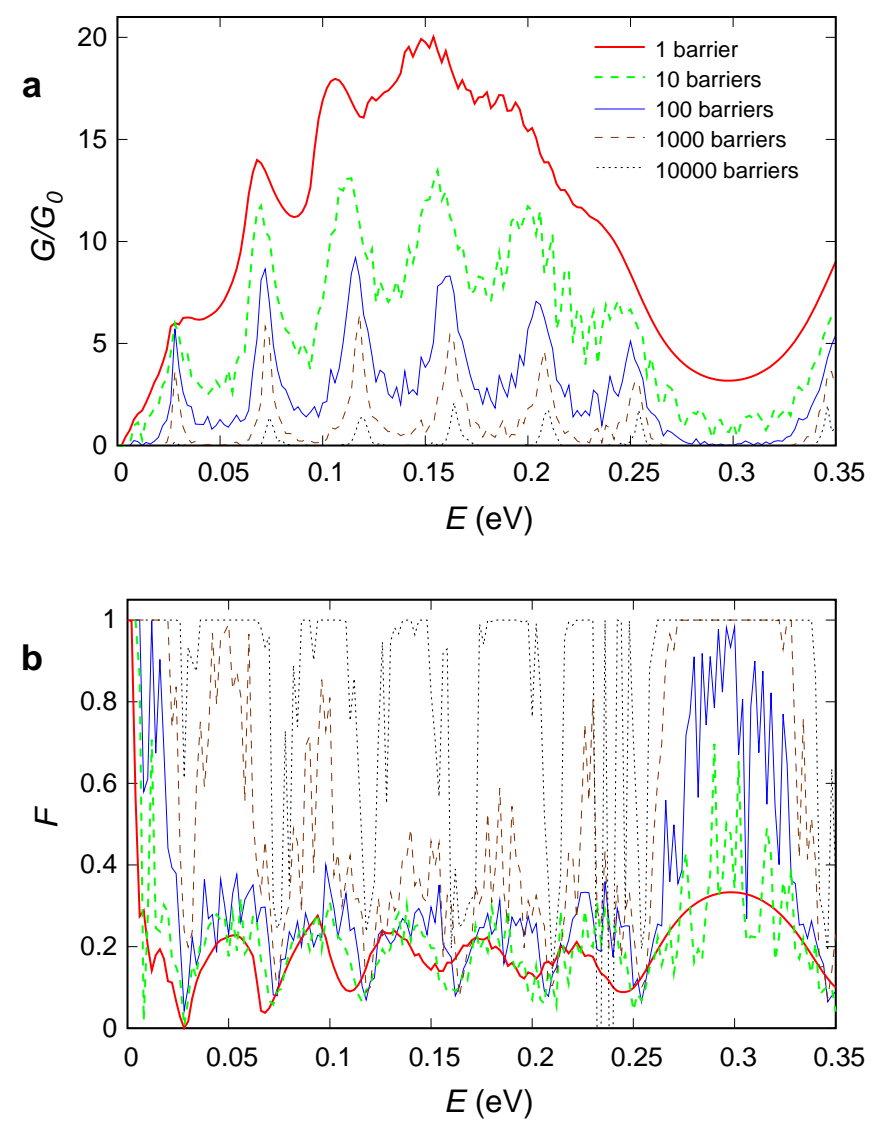

FIG. 2. Behavior, as a function of the injection energy $E$, of the normalized conductance $G / G_{0}$ (a) and of the Fano factor $F$ (b) for the semiconducting ribbon $(N=1626)$ with a potential consisting of a series of identical unevenly spaced rectangular barriers.

conductance behavior that we will discuss in the following is still visible for temperatures up to a few tens of Kelvin.

The initial simulations have been performed for a semiconducting ribbon, containing $N=1626$ dimer lines between the lower and the upper edge, with a potential consisting of a series of identical barriers, with a rectangular profile, each one $40 \mathrm{~nm}$ long and $0.3 \mathrm{eV}$ high. Using a linear congruential generator, we have randomly chosen each inter-barrier distance $d$, with a uniform probability distribution, between $150 \mathrm{~nm}$ and $250 \mathrm{~nm}$.

We have repeated the simulations for several numbers of cascaded barriers. For each set of barriers, we have computed the conductance and the Fano factor behavior as a function of the injection energy $E$. More in detail, for each value of $E$ we have averaged (in the way described in the previous section) the results obtained for 5 energy values uniformly distributed in a $0.4 \mathrm{meV}$ wide interval centered around $E$ (in our simulations 5 values are sufficient, due to the limited variability of the results in this energy interval). Keeping the potential profile fixed and varying the injection energy is equivalent to keeping the injection energy fixed and shifting the potential profile (in the opposite direction). Therefore, this simulation corresponds also to maintaining the Fermi levels of the left and right contacts (differing by $0.4 \mathrm{meV}$ ) constant, while 
shifting the potential profile through the electrostatic action of a bottom gate located underneath the entire device (we remember that the barriers are instead induced by a series of top gates: Fig. 1). The results are shown in Fig. 2(a) and Fig. 2(b) for the conductance $G$ (normalized with respect to the conductance quantum $\left.G_{0}=2 e^{2} / h\right)$ and for the Fano factor $F$, respectively.

The behavior obtained for a single barrier agrees with that reported in the literature ${ }^{34}$. The conductance starts from a zero value for $E=0$, because for this energy the density of states outside the barrier vanishes (no mode propagates for $E=0$ in a semiconducting ribbon). As $E$ is increased, the average behavior of the conductance first increases and then decreases, as an effect of the evolution of the density of states outside and inside the barrier. Superimposed to this average behavior, there are oscillations, with several local maxima and minima. Finally, the conductance reaches a nonzero minimum value when the energy $E$ equates the height of the potential barrier (and the density of states inside the barrier vanishes). Such a nonzero conductance minimum, characteristic of graphene, is an effect of the evanescent modes propagating through the barrier and is also associated to a $1 / 3$ value of the Fano factor ${ }^{27}$. This value (characteristic of the diffusive transport regime) has been attributed to the presence of a Zitterbewegung motion in graphene, which gives rise to a diffusive-like behavior ${ }^{27}$.

Increasing the number of cascaded identical barriers, the conductance drops for all the energies, but the difference between the local minima and the local maxima strongly increases, i.e. the device becomes more selective in energy. Finally, for a rather large number of barriers the conductance vanishes and a strongly localized regime is reached. Regarding the Fano factor, increasing the number of barriers we observe a more irregular behavior as a function of $E$, which reflects the more complex interference pattern in the device. Finally, when transport becomes localized, i.e. for a sufficiently large number of barriers, the Fano factor approaches $F=1$ (no shot noise suppression). Indeed, this is a characteristic feature of the localized regime. While in general Pauli exclusion introduces correlations between charge carriers, which generate deviations in the shot noise power spectral density from the value $2 e I$, when the transmission vanishes the different transition events actually become uncorrelated and the full value of $2 e I$ is recovered.

In order to better understand the observed behavior, we have analyzed the effect of the series of barriers on the single modes propagating through the device. In this case, the barriers, being orthogonal to the ribbon edges, do not introduce any mixing among the different modes. Therefore, each mode transmits across the ribbon independently of the others and the transmission matrix is diagonal, with each element on the diagonal corresponding to the transmission of a single mode.

In analogy with what happens in unconfined graphene, we evaluate the angle of incidence of each mode against the barriers in the following way. Outside the barriers, we can write the generic $i$-th mode in the form $\chi_{i}(y) \exp \left(i \kappa_{x i} x\right)$. We define $\kappa_{x i}$ and $\kappa_{y i}$ the components (in the transport and transverse di-
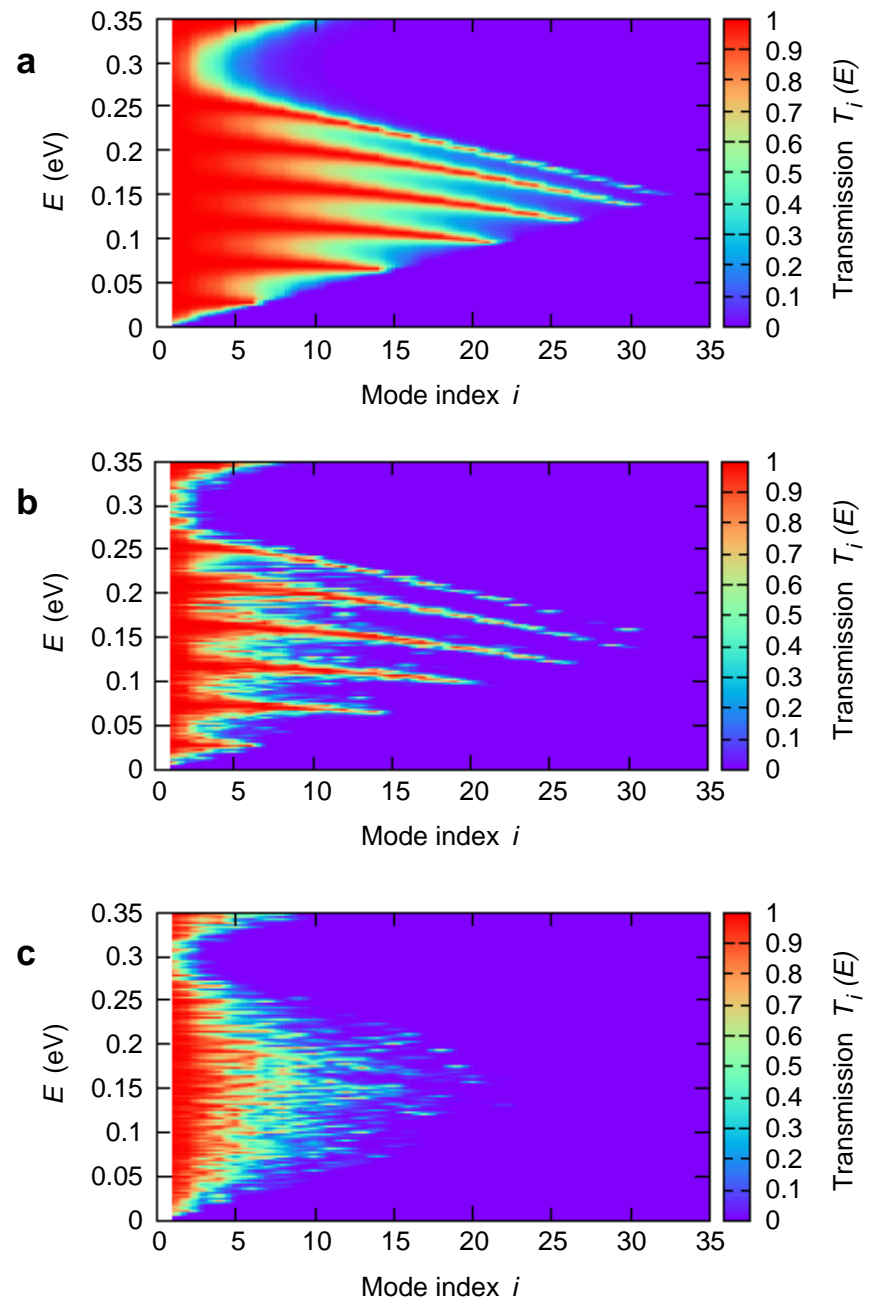

FIG. 3. Plot of the mode transmission (resulting from our numerical simulations) as a function of the mode index $i$ and of the injection energy $E$, for the considered semiconductor ribbon with a single rectangular barrier (a), the cascade of 10 identical unevenly spaced rectangular barriers (b), and the cascade of 10 different unevenly spaced rectangular barriers (c).

rections, respectively) of the wave vector (measured from the Dirac point) of the $i$-th mode outside the barriers. Exploiting the dispersion relation (valid in the regions with zero potential) $E= \pm \hbar v_{F} \sqrt{\kappa_{x i}^{2}+\kappa_{y i}^{2}}$ (where $\hbar$ is the reduced Planck constant and $v_{F}$ is the Fermi velocity of graphene), we define the modulus of the angle of incidence of the $i$-th mode against the barriers as

$$
\left|\theta_{i}\right|=\arctan \left(\left|\frac{\kappa_{y i}}{\kappa_{x i}}\right|\right)=\arctan \left(\frac{\sqrt{\left(E /\left(\hbar v_{F}\right)\right)^{2}-\kappa_{x i}^{2}}}{\left|\kappa_{x i}\right|}\right) .
$$

For each fixed value of $E$, we order the modes by increasing $\left|\theta_{i}\right|$, i.e. by decreasing $\left|\kappa_{x i}\right|$ (or increasing $\left|\kappa_{y i}\right|$ ). Therefore, the lowest modes are those nearest to the condition of orthogonality to the barriers.

In Fig. 3(a) and (b) we report a plot of the transmission 
(resulting from our numerical simulations) of each mode as a function of the mode index $i$ and of the injection energy $E$, for the cases of a single barrier (a) and of ten cascaded identical barriers (b). As we are going to discuss, this behavior can be explained in terms of Klein tunneling and of resonant tunneling through the quasi-localized states in the barriers.

First of all, we observe that the transmission is larger for low-order modes, while decreases increasing the mode index (and thus increasing the incidence angle $\left|\theta_{i}\right|$ ). This is an effect of Klein tunneling: modes impinging closer to orthogonality against the barriers experience a larger transmission. Therefore, a series of tunnel barriers could be used as a mode filter: after a certain number of barriers the highest-order modes have substantially disappeared and only the modes with the lowest incidence angle survive, although with reduced amplitude. This could be useful whenever it is necessary to perform a selection of the modes propagating through the graphene structure. For example, some devices (such as that proposed in Refs. ${ }^{70,71}$, which is based on quantum interference) work well in the presence of a small and selected number of propagating modes. Therefore, an implementation in graphene of these devices would benefit from the upstream presence of a mode-filtering section made up of a series of tunnel barriers.

In this case, we notice that increasing the number of barriers also the transmission of the lowest-order mode (the mode with $i=1$ ) decreases, until it disappears for a large enough number of barriers. Indeed, in a semiconducting ribbon no mode with $\kappa_{y i}=0$ exists. More in detail, the Dirichlet boundary conditions at the ribbon edges limit the allowed values of the total transverse wave vector to multiples of $\pi / W$. The Dirac point has ordinate (in the reciprocal space) $4 \pi /(3 a)$, while $W=(N+1) a / 2$ ( $a$ is the graphene lattice constant and $N$ is the number of dimer lines across the ribbon width). Therefore, in a semiconducting ribbon (where $N$ is not equal to $3 M-1$, with $M$ an integer) the minimum value of the modulus $\left|\kappa_{y i}\right|$ of the difference between the transverse component of the allowed wave vectors and the ordinate of the Dirac point is equal to $\pi /(3 W)$. More in general, the $i$-th mode has

$$
\left|\kappa_{y i}\right|=\ell \frac{\pi}{3 W}, \text { with } \ell=i+\left\lfloor\frac{i-1}{2}\right\rfloor=1,2,4,5,7, \ldots
$$

(i.e., the set of values $\ell$ is obtained by removing the multiples of 3 from the sequence of the natural numbers). Since no mode exists with $\kappa_{y i}=0$ (i.e., orthogonal to the barriers), crossing the barriers all the modes (including the lowest one) experience attenuation (although in different amounts) and, if we sufficiently increase the number of barriers, vanish.

Beyond this average behavior, we observe a dependence on the energy of the transmission of the single modes (a dependence that we have already observed in the overall device conductance, reported in Fig. 2(a)). This feature can be explained in terms of resonant tunneling with the states quasi-localized inside each of the barriers.

Let us consider a single $L_{B}$ long and $U_{B}$ high barrier. We indicate with $\kappa_{x}$ and $\kappa_{y}$ the components (in the direction orthogonal and parallel to the barrier, respectively) of the wave vector in the regions outside the barrier. In a similar way, we define $\kappa_{x}^{\prime}$ and $\kappa_{y}^{\prime}$ as the components of the wave vector inside

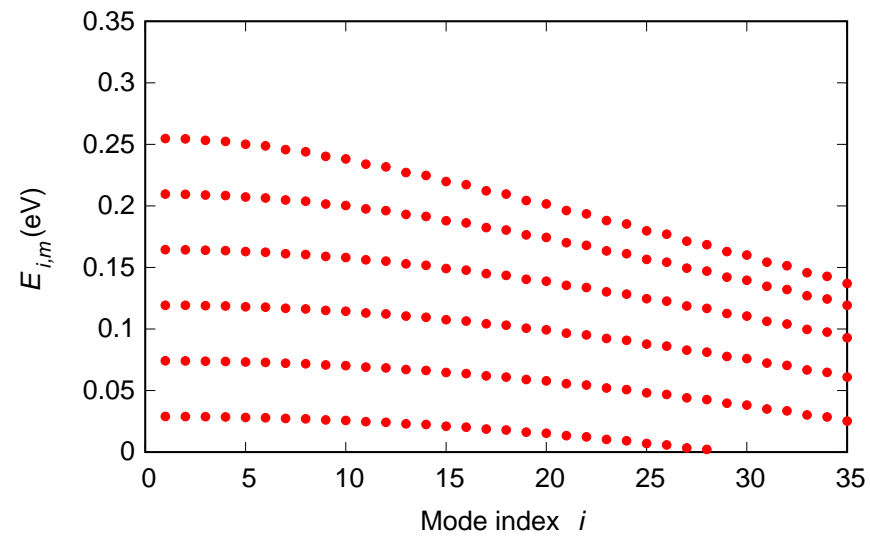

FIG. 4. Energies $E_{i, m}$ (obtained from Eq. (4) and Eq. (3)) of the states quasi-localized inside a tunnel barrier in the considered semiconducting ribbon, plotted as a function of the mode index $i$.

the tunnel barrier. Let us focus on the range of energies between 0 and $U_{B}$. Outside the barrier, the carriers in the $i$-th mode have an energy $E=\hbar v_{F} \sqrt{\kappa_{x i}^{2}+\kappa_{y i}^{2}}$. The barrier actually represents an $L_{B}$-long potential well for the hole states ${ }^{34}$. Therefore, inside the barrier quasi-confined states form, with discrete values of the longitudinal component of the wave vector, given by $\kappa_{x m}^{\prime}=m \pi / L_{B}$ (with $m$ an integer). Moreover, assuming sufficiently regular barrier edges, the component of the wave vector parallel to the barrier does not change when the carriers pass from the region outside the barrier to the region inside the barrier and vice versa ${ }^{72}$ : the $i$-th mode outside the barrier is coupled only to the $i$-th mode inside the barrier and $\kappa_{y i}^{\prime}=\kappa_{y i}=\ell \pi /(3 W)$. As a consequence, the quasi-bound states inside the barrier have the following energies:

$$
\begin{aligned}
E_{i, m} & =U_{B}-\hbar v_{F} \sqrt{{\kappa_{x m}^{\prime}{ }^{2}+\kappa_{y i}^{\prime 2}}^{2}}= \\
& =U_{B}-\hbar v_{F} \sqrt{\left(m \pi / L_{B}\right)^{2}+(\ell \pi /(3 W))^{2}},
\end{aligned}
$$

with $\ell$ related to $i$ by Eq. (3). Focusing on the $i$-th mode, when the carriers outside the barrier impinge against it with an energy $E$ identical to $E_{i, m}$ (the energy of one of the states quasilocalized inside the barrier), the transmission has a maximum. As we see in Fig. 4, where we plot these values $E_{i, m}$ as a function of the mode index $i$, these energies fit well the energies for which the transmission maxima are observed in Fig. 3: the generic mode $i$ experiences a maximum transmission for the energy values $E=E_{i, m}$. Therefore, the barrier operates an energy filtering on the wave function impinging against it.

Since we are considering the case of identical barriers, i.e. of barriers with the same length $L_{B}$ and height $U_{B}$, the energy values $E_{i, m}$ for which the transmission is maximum are the same for all the barriers. Therefore, any additional barrier further refines the energy selection already operated by the previous ones. As a consequence, increasing the number of barriers, we observe (besides the general reduction of transmission deriving from the increased backscattering) a larger energy filtering effect, i.e., an enhanced ratio of the local maxima to the local minima of the conductance as a function of energy. 


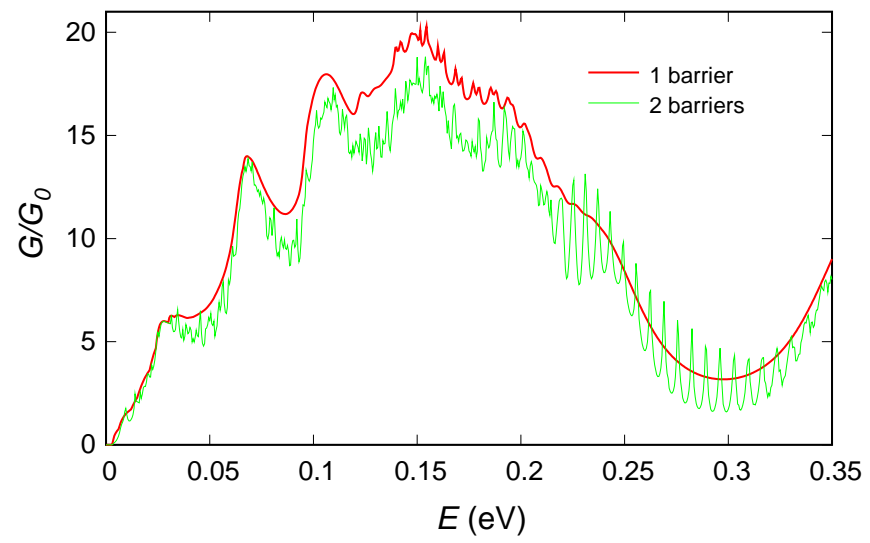

FIG. 5. Behavior, as a function of the injection energy $E$, of the normalized conductance $G / G_{0}$ for the considered semiconducting ribbon, with a potential consisting of one or two identical rectangular barriers.

This energy filtering effect could be useful for example for the implementation of low-noise devices ${ }^{73}$, or more in general of devices which require or take advantage from the injection of charges with a limited number of well-defined energies.

A first estimation of the position of the peaks observed in the behavior of the total conductance as a function of energy (reported in Fig. 2(a)) can be made focusing on the modes nearest to the orthogonality condition (due to Klein tunneling, these modes, characterized by a small $\kappa_{y i}^{\prime}$, have the highest transmission). The energies $E_{m}$ at which these modes experience resonant tunneling can be obtained from Eq. (4) disregarding $\kappa_{y i}^{\prime}$ and considering different values of the integer $m$ (corresponding to different states quasi-localized inside the barriers): $E_{m}=U_{B}-\hbar v_{F}\left(m \pi / L_{B}\right)$.

Since, through the quantity $I=G V$ (the quantity $V$ being the voltage applied between the device contacts), the conductance appears at the denominator of the Fano factor, this effect has a direct impact also on the behavior of the Fano factor as a function of energy (as we see in Fig. 2(b)).

We notice that, since we are considering the case of different inter-barrier distances, the resonant tunneling effects deriving from the alignment of the injection energy with the energies of the states quasi-localized in the regions between adjacent barriers (which actually represent wells for electrons) can be disregarded. Indeed, the energies of these quasilocalized states are not the same for inter-barrier regions with different lengths. The difference between the inter-barrier distances makes it also possible to disregard the formation of new Dirac points that has been reported in the literature for the case of evenly spaced barriers. The only exception is the case of two cascaded barriers, where the resonance with the states quasi-localized in the region between the barriers gives rise to further oscillations, superimposed onto those deriving from the resonance with the states quasi-localized inside the barriers (see Fig. 5). In our case, the oscillations due to the resonance with the states quasi-localized in the inter-barrier regions have an energy separation smaller than those due to the resonance with the states quasi-localized inside the barri-

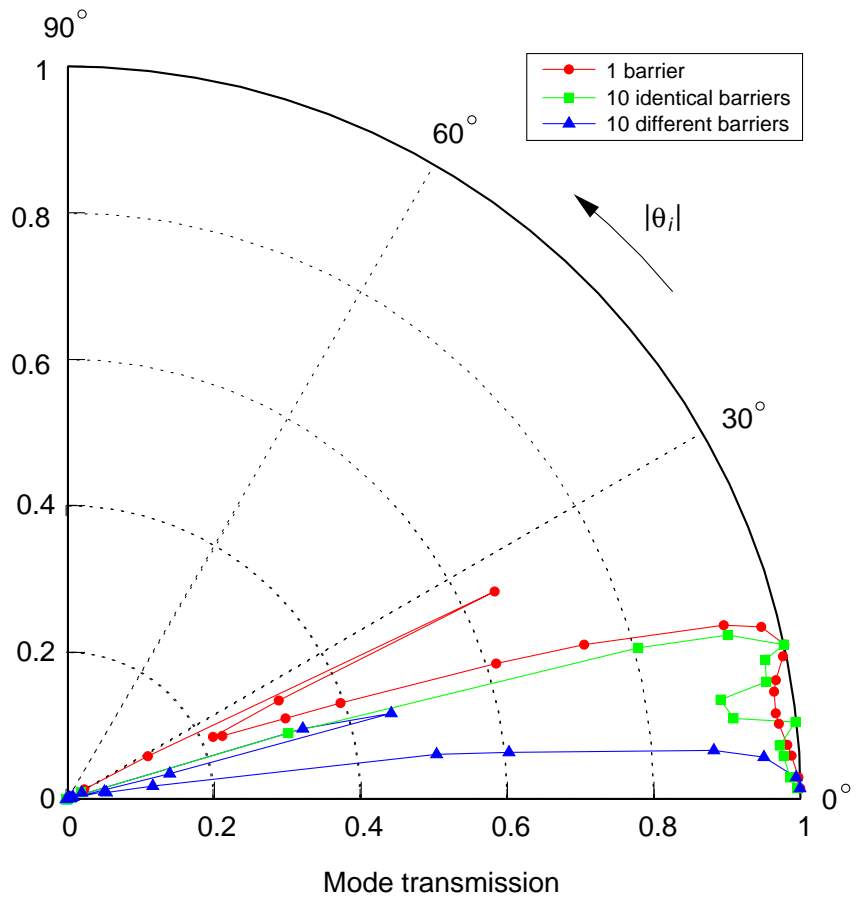

FIG. 6. Polar plot of the mode transmission as a function of the modulus of the incidence angle $\left|\theta_{i}\right|$ (represented in the figure by the counterclockwise angle from the horizontal axis) for the semiconducting ribbon $(N=1626)$ with: a single rectangular barrier (red dots), 10 unevenly spaced identical rectangular barriers (green squares) and 10 unevenly spaced different rectangular barriers (blue triangles).

ers, since the distance between the barriers is larger than the length of each barrier.

In Fig. 6, on a polar plot, we report the transmission for the various modes as a function of the modulus of their incidence angle, for a fixed energy (in our case $E=0.2 \mathrm{eV}$ ). The results for a single barrier and for 10 unevenly spaced identical barriers are represented with red circles and green squares, respectively, and can be obtained from the sections, taken for the considered energy $E$, of the maps reported in Fig. 3. This plot provides further graphical evidence for the phenomenon of Klein tunneling (modes closer to the condition of orthogonality experience larger transmission), with some oscillations due to resonant tunneling, since for a fixed value of $E$ a discrete number of modes exists for which the resonant tunneling condition $E \approx E_{i, m}$ is reached.

We have then simulated the behavior of the same semiconducting ribbon when the barriers are not identical. In particular, we have considered $0.3 \mathrm{eV}$ high rectangular barriers, differing for their length, which we have randomly chosen (with a uniform probability distribution) between $5 \mathrm{~nm}$ and $75 \mathrm{~nm}$. All the other parameters are left identical to those for the previous calculations. The results are reported in Fig. 3(c), Fig. 6 (with blue triangles), and Fig. 7. Also in this structures, we observe a larger transmission for low-order modes, as an effect of Klein tunneling. Regarding resonant tunneling, instead, in this case the energies of the states quasi-localized inside one barrier differ from those of the other barriers because the barriers have different lengths $L_{B}$. Therefore, energies for 

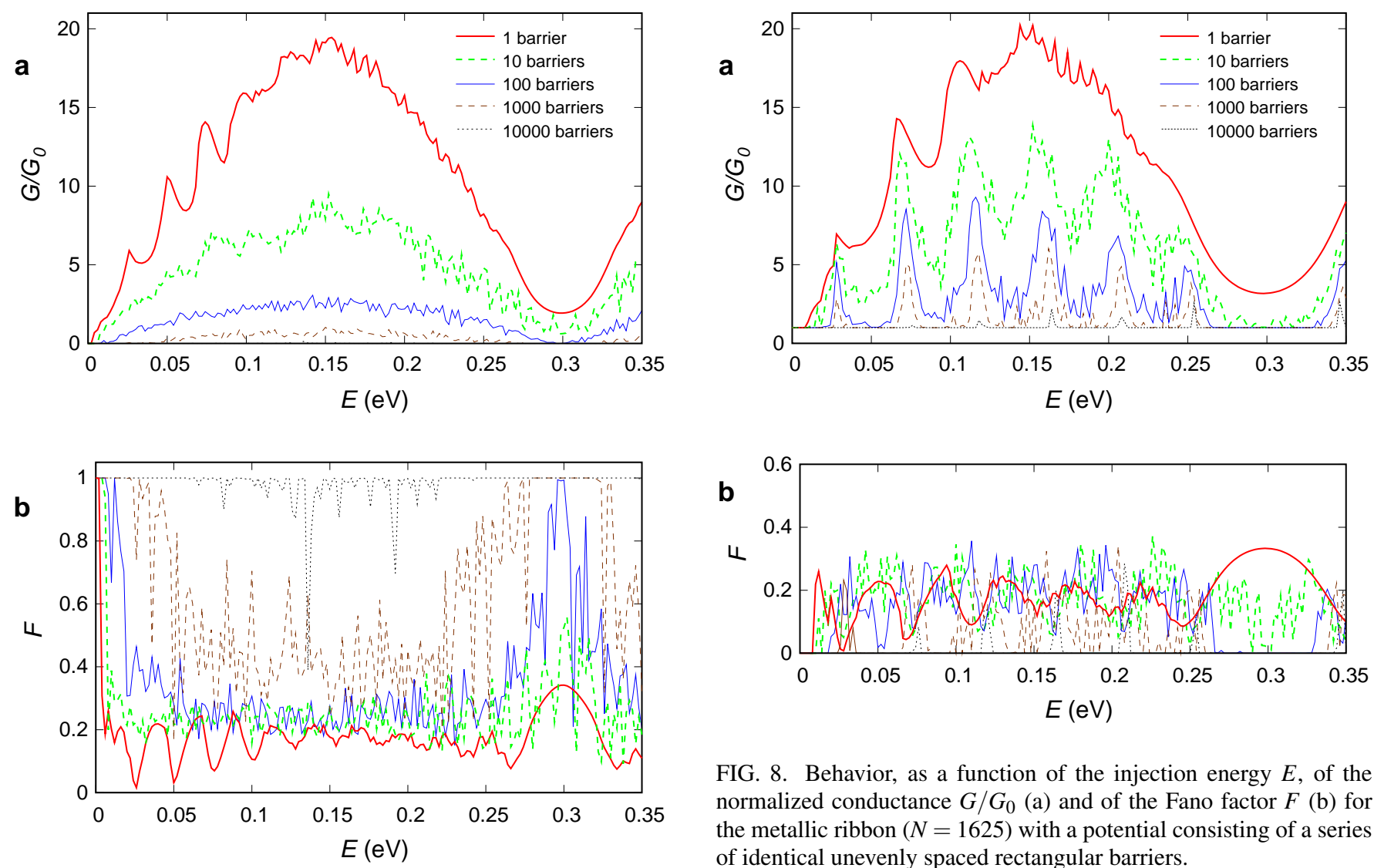

FIG. 8. Behavior, as a function of the injection energy $E$, of the normalized conductance $G / G_{0}$ (a) and of the Fano factor $F$ (b) for the metallic ribbon $(N=1625)$ with a potential consisting of a series of identical unevenly spaced rectangular barriers.

FIG. 7. Behavior, as a function of the injection energy $E$, of the normalized conductance $G / G_{0}$ (a) and of the Fano factor $F$ (b) for the semiconducting ribbon $(N=1626)$ with a potential consisting of a series of different unevenly spaced rectangular barriers.

which the impinging carriers experience maximum (or minimum) transmission across all the barriers do not exist. As a consequence, the behavior of the conductance as a function of energy is smoothed out, without any evidence of local maxima or minima between $E=0$ and $E=U_{0}$ (Fig. 7(a)). This clearly affects also the behavior of the Fano factor as a function of energy (Fig. 7(b)), the maps of the transmission as a function of the mode index and of the energy (Fig. 3(c)), and the polar plots of the transmission as a function of the modulus of the incidence angle (blue triangles in Fig. 6): for more than one barrier the effect of resonant tunneling actually disappears.

We have then analyzed the case of a metallic graphene ribbon with a series of unevenly spaced identical potential barriers, with a rectangular profile. In this case the ribbon has $N=1625$ dimer lines across its width and thus $N$ is equal to $3 M-1$, with $M$ an integer $(M=542)$.

In Figs. 8-11 we report results analogous to those previously shown for the case of the semiconducting ribbon. In Fig. 8 we plot the behavior of the conductance and of the Fano factor as a function of the injection energy $E$, in Fig. 9(a) and (b) we report the maps of the mode transmission as a function of the mode index $i$ and of the injection energy $E$, and in Fig. 11 we represent a polar plot of the transmission of the modes as a function of the modulus of their incidence angle

for a fixed injection energy $E=0.2 \mathrm{eV}$.

Also in this case, modes with a smaller angle of incidence against the barriers experience a much larger transmission, due to Klein tunneling. We observe also the effect of resonant tunneling, with a transmission that strongly depends on the injection energy (and in particular on the presence or absence at that energy of a state quasi-localized inside the barriers). Again, the dependence on energy increases with the number of identical barriers, as a consequence of the combined filtering action of the barriers.

The difference with respect to the case of the semiconducting ribbon is that in this case, being $N=3 M-1$, the modulus $\left|\kappa_{y i}\right|$ of the difference between the allowed values of the transverse wave vector (enforced to multiples of $\pi / W$ by the Dirichlet boundary conditions at the ribbon edges) and the ordinate of the Dirac point takes on the following values:

$$
\left|\kappa_{y i}\right|=\ell \frac{\pi}{3 W}, \text { with } \ell=3\left\lfloor\frac{i}{2}\right\rfloor=0,3,3,6,6, \ldots
$$

(which replaces in this case Eq. (3)). Substituting this expression into Eq. (4) we obtain the energies for which the transmission for the different modes reaches its local maxima. These values, reported in Fig. 10 as a function of the mode index $i$, fit well the energies for which we have found the transmission maxima (see Fig. 9). In particular, the lowest mode (with $i=1$ ) has $\kappa_{y i}=0$. This means that this mode propagates also for $E=0$ (and actually in our simulations $G=G_{0}$ for $E=0$, as shown in Fig. 8(a)) and also that it has $\theta_{i}=0$, i.e. this 

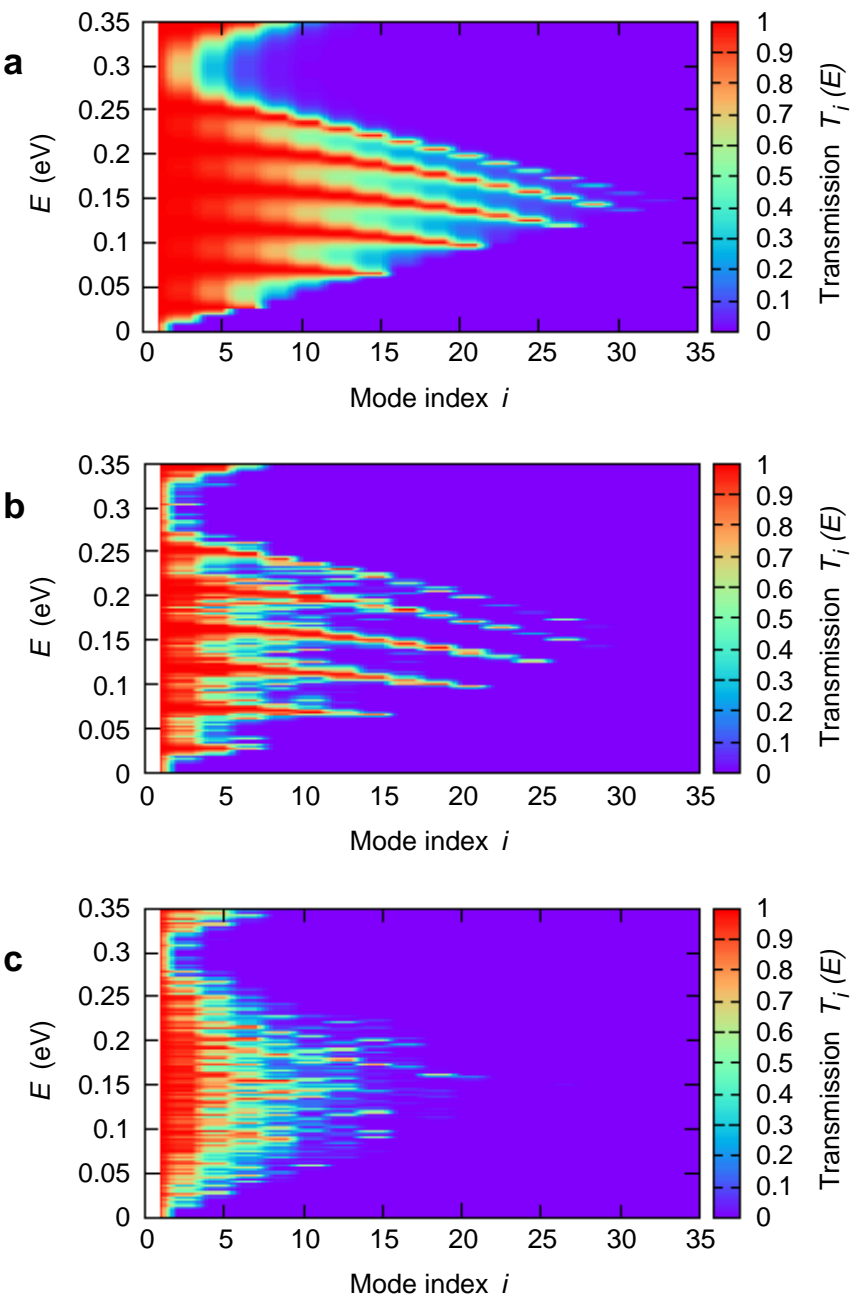

FIG. 9. Plot of the mode transmission as a function of the mode index $i$ and of the injection energy $E$, for the metallic ribbon $(N=1625)$ with a single rectangular barrier (a), the cascade of 10 identical unevenly spaced rectangular barriers (b), and the cascade of 10 different unevenly spaced rectangular barriers (c).

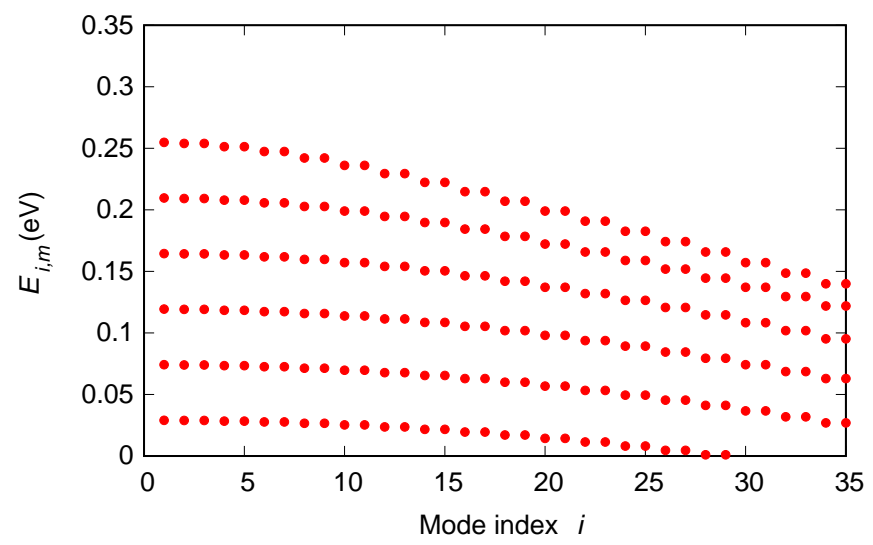

FIG. 10. Energies $E_{i, m}$ (obtained from Eq. (4) and Eq. (5)) of the states quasi-localized inside a tunnel barrier in the considered metallic ribbon, plotted as a function of the mode index $i$.

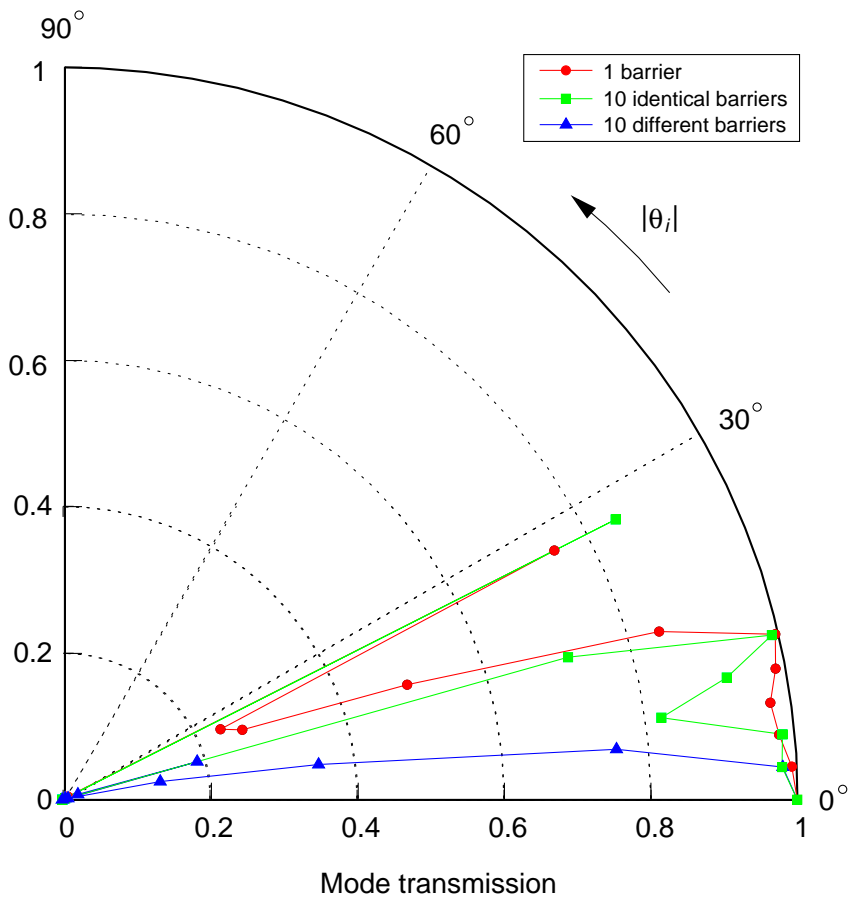

FIG. 11. Polar plot of the mode transmission as a function of the modulus of the incidence angle $\left|\theta_{i}\right|$ for the metallic ribbon $(N=$ 1625 ) with: a single rectangular barrier (red dots), 10 unevenly spaced identical rectangular barriers (green squares) and 10 unevenly spaced different rectangular barriers (blue triangles).

mode impinges orthogonally against the barriers. As a consequence of Klein tunneling, this mode does not experience any backscattering and is transmitted perfectly by the device, for any number of barriers. Therefore, sufficiently increasing the number of barriers, one propagating mode remains and ballistically transmits across the structure, giving a total normalized conductance $G / G_{0}$ equal to 1 (see Fig. 8(a)). As a consequence, the Fano factor approaches zero (see Fig. 8(b)), which is the value characteristic of perfect transmission. Indeed, in this case the effect of Pauli exclusion regularizes the transport dynamics across the device (because each state can be occupied by one electron at a time and there is a finite number of available states) and shot noise vanishes ${ }^{74}$. Therefore, the behavior of the Fano factor is heavily dependent on the exact width of the ribbon, as a result of a significant change in the transport regime.

In Fig. 9(c), Fig. 11 (with blue triangles), and Fig. 12, we report the results for the same metallic ribbon when the barriers are not identical and in particular differ for their length, randomly chosen between $5 \mathrm{~nm}$ and $75 \mathrm{~nm}$. The conclusion is analogous to that reached in the case of the semiconducting ribbon: when the cascaded barriers differ, the effect of resonant tunneling disappears (while Klein tunneling is still present).

It is also interesting to consider what happens around $E=0$ in the cases we have reported. For semiconducting ribbons, no propagating mode exists for $E=0$ : therefore, the conductance is zero and the Fano factor is 1. Instead, for metallic ribbons, for $E=0$ the lowest mode can propagate, giving rise 

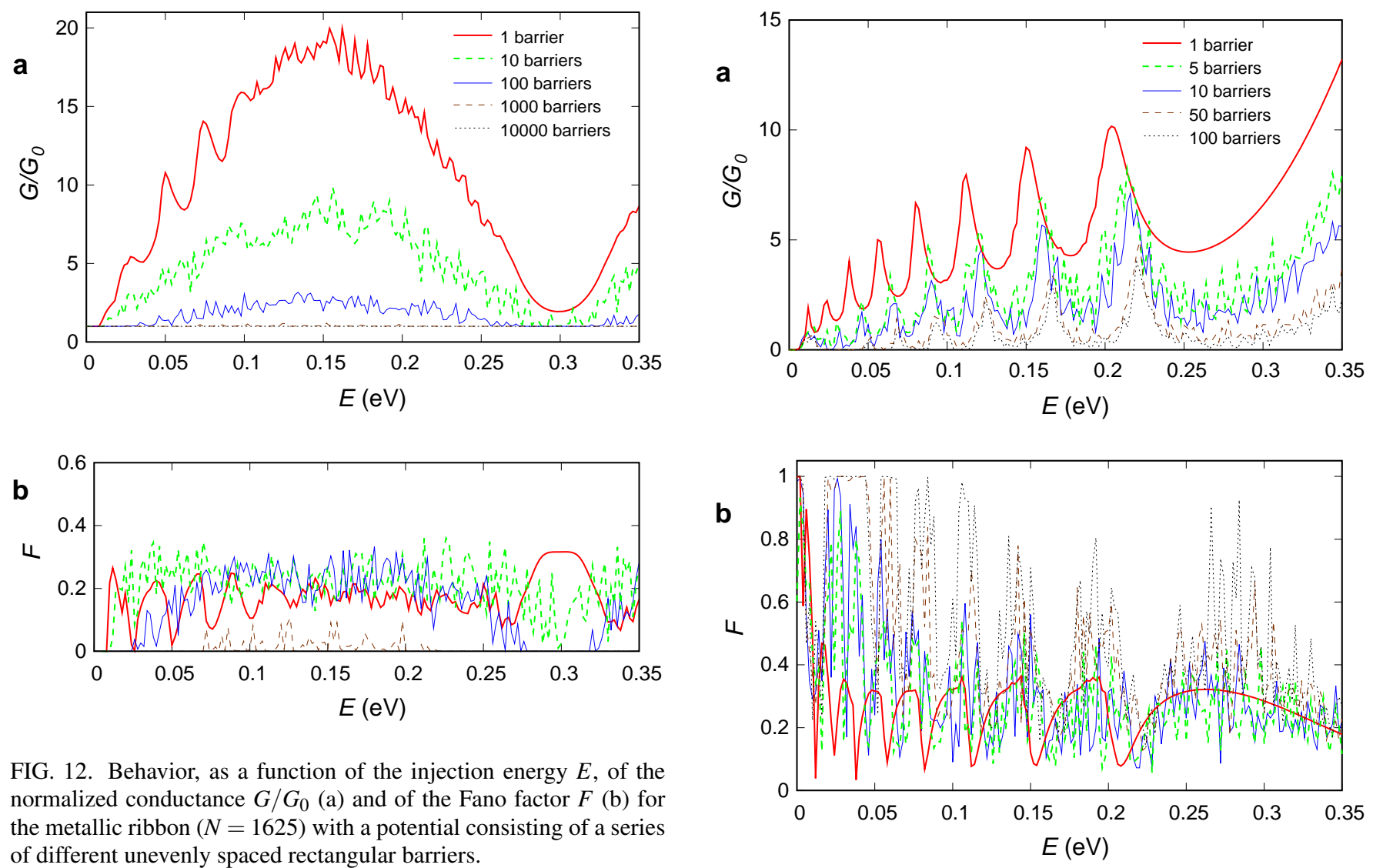

FIG. 12. Behavior, as a function of the injection energy $E$, of the normalized conductance $G / G_{0}$ (a) and of the Fano factor $F$ (b) for the metallic ribbon $(N=1625)$ with a potential consisting of a series of different unevenly spaced rectangular barriers.

to a nonzero conductance $G=G_{0}$ and to a vanishing Fano factor. It can be interesting to compare these results with what has been observed for a single barrier by Tworzydło et al. ${ }^{27}$. Indeed, for $E=0$ the overall device extending from the left lead to the right one can be seen as a single long barrier with respect to the regions with negative potential that represent the contacts. In this case the length of the overall equivalent barrier (i.e. the length of the device) is not much smaller than the width of the ribbon, Therefore, we are exactly in the conditions in which Tworzydło et al. predicted a behavior different for metallic and semiconducting ribbons, with conductance zero for semiconducting ribbons (for structures with a length much larger than the width, the evanescent modes are not able to give a nonzero contribution) and nonzero for metallic ones (where a propagating mode exists for zero energy), in agreement with our results. The presence of barriers inside the device does not alter these results: neither in the semiconducting ribbons (where the barriers represent a further obstacle to the transmission, which is zero already in the their absence), nor in the metallic ribbons (where the mode with zero energy, having $\kappa_{y i}=0$, is orthogonal to the barriers and thus does not experience any scattering from the barriers themselves). Note that the situation is different from what is observed for $E=U_{0}$ for the case of a single barrier: in that case, the barrier that we have to consider is the real barrier, which has a length much smaller than the ribbon width. In those conditions, Tworzydło et al. predict a nonzero conductance (equal to $\left.\left(4 e^{2} /(\pi h)\right)\left(W / L_{B}\right)\right)$ and a $1 / 3$ Fano factor both for semi-

FIG. 13. Behavior, as a function of the injection energy $E$, of the normalized conductance $G / G_{0}$ (a) and of the Fano factor $F$ (b) for the semiconducting ribbon $(N=1626)$ with a potential consisting of a series of identical unevenly spaced Lorentzian barriers.

conducting and for metallic ribbons, in agreement with our numerical results.

We have performed analogous simulations on barriers that are not rectangular. We report the results for a semiconducting ribbon with $N=1626$ (see Figs. 13 and 14) and for a metallic ribbon with $N=1625$ (see Fig. 15) considering barriers with a Lorentzian profile, with a $0.3 \mathrm{eV}$ peak. The half-width at halfmaximum of the barriers is equal to $20 \mathrm{~nm}$ in the case of identical barriers, while it is a random value extracted with a uniform probability distribution between $2.5 \mathrm{~nm}$ and $37.5 \mathrm{~nm}$ in the case of different barriers. For the inter-barrier distances we have chosen values similar to those considered in the simulations for rectangular barriers. Due to the longer computational times required by the simulation of ribbons with smoothly varying potential barriers, in this case we have considered a smaller number of cascaded barriers.

In Figs. 13 and 15 we show the behavior of the conductance and of the Fano factor as a function of the injection energy for different numbers of Lorentzian barriers in the case of identical barriers in a semiconducting and in a metallic ribbon, respectively. The results obtained in the case of different barriers differ from these for the absence of strong oscillations with energy.

Apart from the values of the energies of the quasi-confined 


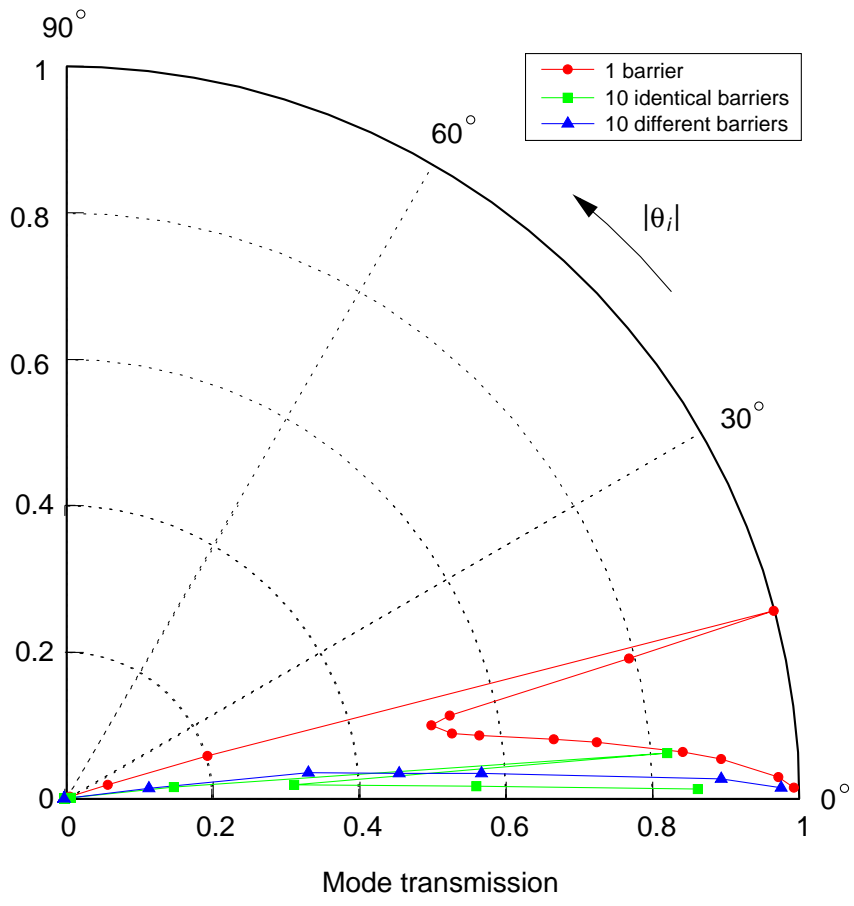

FIG. 14. Polar plot of the mode transmission as a function of the modulus of the incidence angle $\left|\theta_{i}\right|$ for the semiconducting ribbon $(N=1626)$ with: a single Lorentzian barrier (red dots), 10 unevenly spaced identical Lorentzian barriers (green squares) and 10 unevenly spaced different Lorentzian barriers (blue triangles).
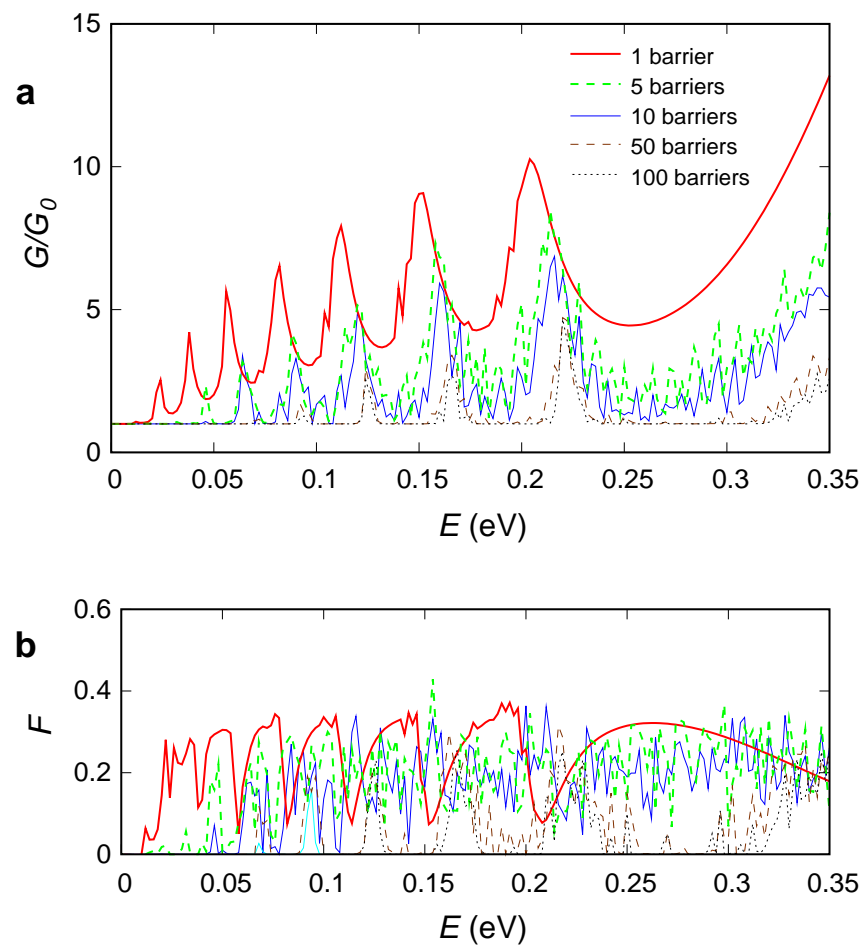

FIG. 15. Behavior, as a function of the injection energy $E$, of the normalized conductance $G / G_{0}$ (a) and of the Fano factor $F$ (b) for the metallic ribbon $(N=1625)$ with a potential consisting of a series of identical unevenly spaced Lorentzian barriers.
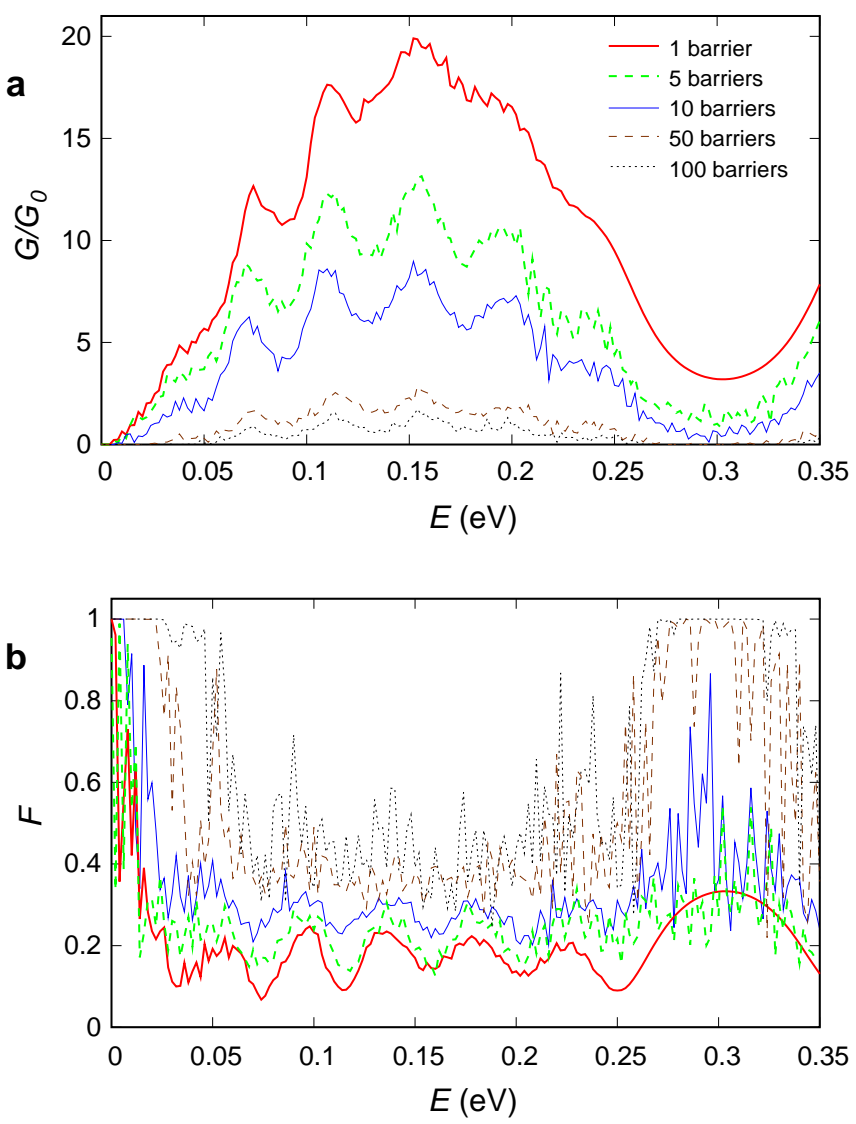

FIG. 16. Behavior, as a function of the injection energy $E$, of the normalized conductance $G / G_{0}$ (a) and of the Fano factor $F$ (b) for the semiconducting ribbon $(N=1626)$ with a potential consisting of a series of identical unevenly spaced rectangular barriers, in the presence of potential disorder.

states inside the barriers, which depend on the specific barrier profiles, we see that the main features observed in the case of rectangular barriers are preserved. However, in this case the oscillations of the conductance and of the Fano factor as a function of energy are much larger: indeed, barriers with a smoother potential profile are known to present a more selective Klein tunneling effect ${ }^{33,75}$. This is confirmed by the polar plot of the mode transmission as a function of the modulus of the incidence angle, that we show in Fig. 14 for the case of a semiconducting ribbon with identical or different unevenly spaced Lorentzian barriers.

In order to assess if our conclusions can survive in a realistic scenario, we have repeated the simulations performed on rectangular barriers adding disorder to the previously considered potential profile. This disorder, which can represent the electrostatic effect of the charged impurities unavoidably present near the graphene ribbon, has been simulated through a superposition of randomly located Gaussian scatterers $^{76}$. We have assumed a scatterer concentration equal to $5 \times 10^{10} \mathrm{~cm}^{-2}$, and for each Gaussian function a half-width at half-maximum of $35 \mathrm{~nm}$ and a random height uniformly distributed between $-10 \mathrm{meV}$ and $10 \mathrm{meV}$ (values which are consistent with a low but still realistic level of disorder). As 

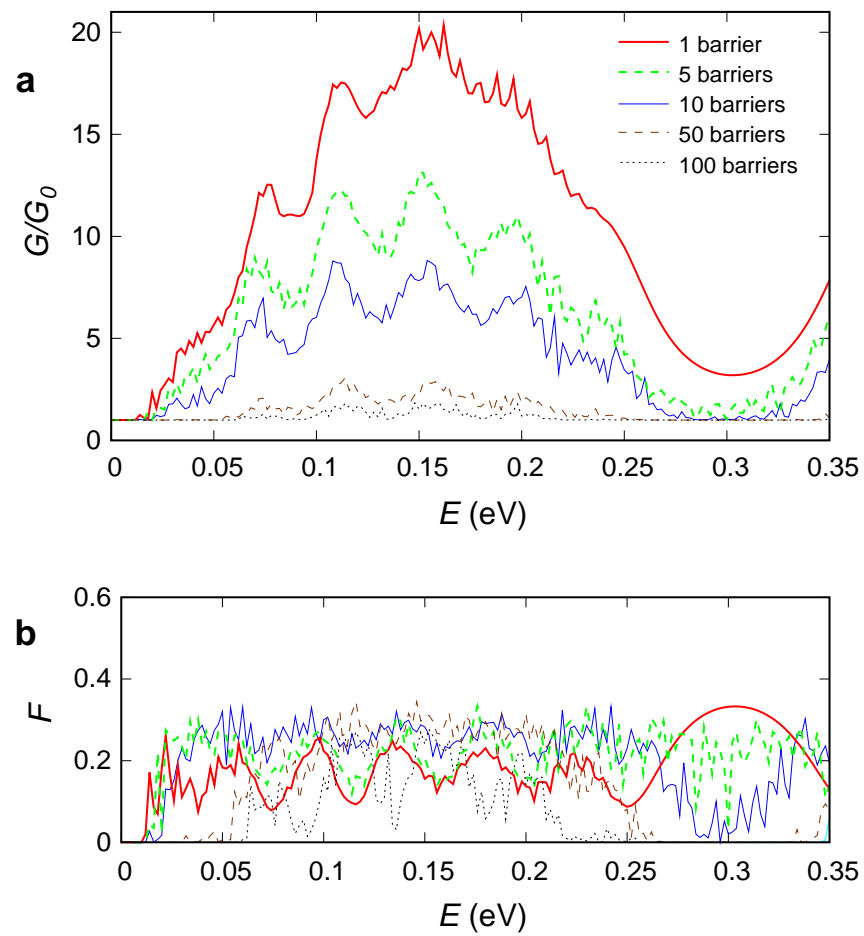

FIG. 17. Behavior, as a function of the injection energy $E$, of the normalized conductance $G / G_{0}$ (a) and of the Fano factor $F$ (b) for the metallic ribbon $(N=1625)$ with a potential consisting of a series of identical unevenly spaced rectangular barriers, in the presence of potential disorder.

we show (for example for the case of identical barriers) in Figs. 16 and 17, in this case the main features obtained in the previous simulations are still clearly visible. Therefore, these effects (that would be washed out by a sufficiently strong potential disorder) should however be observable in a practical experiment on very clean samples.

Finally, we have performed a simulation for barriers that are at an angle different from $90^{\circ}$ with respect to the ribbon edges, although all at the same angle. In particular, we have considered $0.3 \mathrm{eV}$ high barriers with a rectangular profile, tilted by $45^{\circ}$ with respect to the ribbon edges. In Figs. 18 and 19 we show the results of our simulations, performed for a semiconducting ribbon with $N=1626$ and for a metallic ribbon with $N=1625$, in the case of identical barrier lengths (analogous considerations are valid in the case of different barriers). The lengths, measured in the $x$ direction, are identical to those considered in the previous simulations. Therefore, as a result of the $45^{\circ}$ tilt, the barriers we have simulated have a thickness of $40 \mathrm{~nm} / \sqrt{2}$, with inter-barrier distances varying from $150 \mathrm{~nm} / \sqrt{2}$ to $250 \mathrm{~nm} / \sqrt{2}$.

Analyzing the behavior of the conductance as a function of energy and comparing it with that observed in the case of barriers orthogonal to the ribbon edges, we observe a larger conductance suppression, besides the presence (already noticed in Ref. ${ }^{56}$ for a single tilted barrier) of a greater number of fine structures in the $G(E)$ curve in the low-energy range (and especially for a small number of barriers).
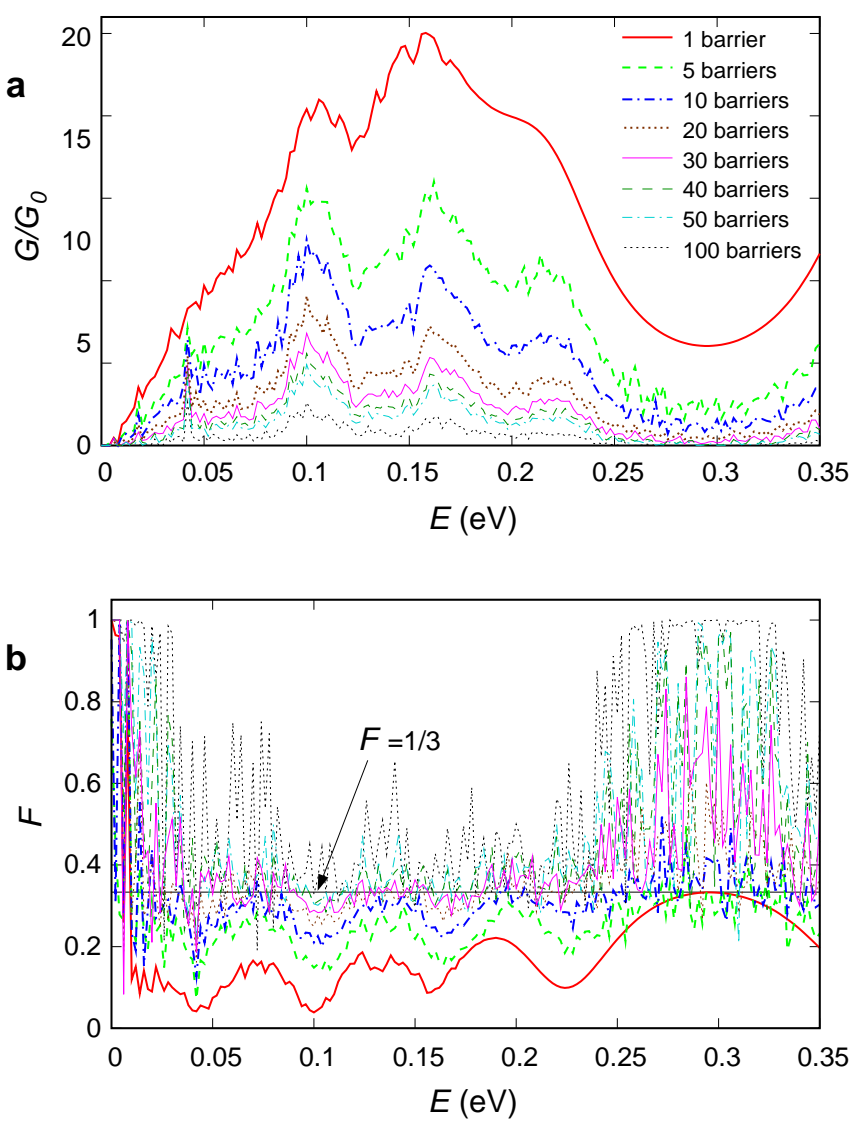

FIG. 18. Behavior, as a function of the injection energy $E$, of the normalized conductance $G / G_{0}$ (a) and of the Fano factor $F$ (b) for the semiconducting ribbon $(N=1626)$ with a potential consisting of a series of identical unevenly spaced rectangular barriers, tilted by a $45^{\circ}$ angle with respect to the ribbon edges.

Apart from this, we still observe features qualitatively similar to those seen in the case of barriers orthogonal to the ribbon edges, with mode-filtering (in particular, note that in the case of a metallic ribbon one transmission mode still passes unaltered through the series of barriers, as it was observed for a single tilted barrier in Ref. ${ }^{56}$ ) and, in case of identical barriers, energy filtering. Indeed, if here we focus our attention on the states that diagonalize the transmission matrix, we observe that the physical phenomena (Klein tunneling and resonant tunneling) which dominated transport for barriers orthogonal to the ribbon edges are still important in the case of tilted barriers.

However, looking at the behavior of the Fano factor as a function of energy (reported in the panels (b) of Figs. 18 and 19) we notice an interesting feature: for a large range of energies the Fano factor approaches $1 / 3$, the value typical of diffusive transport. The presence of a diffusive transport regime is confirmed by the fact that we have observed a linear behavior of the resistance as a function of the length of the region with potential barriers.

In general, diffusive transport takes place when the device length is much larger than the mean free path and much smaller than the localization length (which is of the order of 

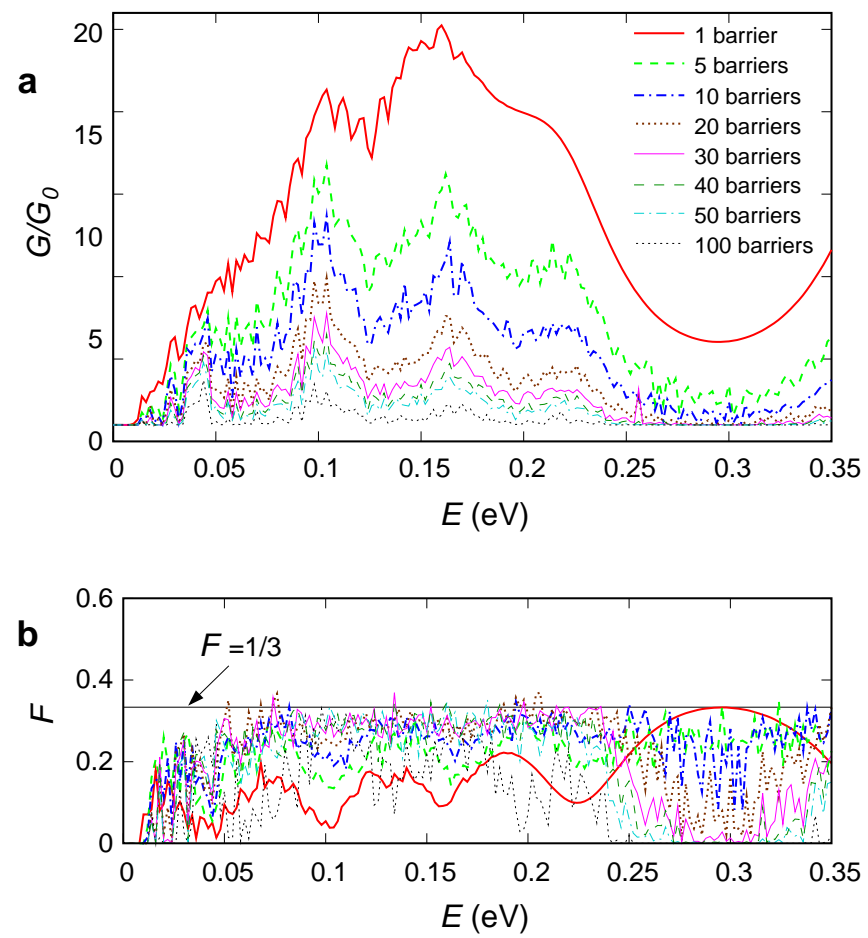

FIG. 19. Behavior, as a function of the injection energy $E$, of the normalized conductance $G / G_{0}$ (a) and of the Fano factor $F$ (b) for the metallic ribbon $(N=1625)$ with a potential consisting of a series of identical unevenly spaced rectangular barriers, tilted by a $45^{\circ}$ angle with respect to the ribbon edges.

the mean free path times the number of propagating modes). When the device length is much smaller than the mean free path, transport is ballistic, while when the device is much longer than the localization length, transport is strongly localized.

However, in the case of barriers orthogonal to the transport direction, the barriers do not introduce any mixing among the modes and thus the different modes independently propagate along the ribbon. Therefore, the system can be considered as a superposition of several single-mode systems, for which the localization length is of the order of the mean free path and thus reaching the diffusive regime is practically impossible. This is, indeed, what we have seen in the simulations with non-tilted barriers, where no trend towards $1 / 3$ has been observed in the behavior of the Fano factor.

Instead, when the barriers are tilted, they introduce mixing among the modes and thus the ribbon, instead of being a collection of single-mode systems, represents a real multimode channel. Therefore, the localization length can be much greater than the mean free path and the diffusive regime (with the $1 / 3$ suppression of the Fano factor) can be reached, as we have indeed observed in our simulations.

The situation is analogous to the one previously investigated in the literature ${ }^{57,58}$ for a series of unevenly spaced barriers in a wire obtained confining the two-dimensional electron gas (2DEG) in a semiconductor heterostructure (for example GaAs/AlGaAs). In that case, for barriers orthogonal to the transport direction a quantum simulation found a transport behavior changing from ballistic to localized as the number of barriers was increased, without experiencing the diffusive regime $^{57}$.

Since the arguments that we have used to explain our numerical results in a graphene ribbon in the presence of tilted barriers are quite general, they should be valid also for a wire in a GaAs/AlGaAs heterostructure. In order to test the validity of this conjecture, we have performed some simulations for a $800 \mathrm{~nm}$ wide wire with a number (varying from 1 to 20) of $45^{\circ}$ tilted rectangular barriers, with a $0.25 \mathrm{eV}$ height and a $0.2 \mathrm{~nm}$ length. The distance between the adjacent barriers has been chosen randomly between $90 \mathrm{~nm}$ and $110 \mathrm{~nm}$. We have considered an injection energy $E=9 \mathrm{meV}$, averaging the results over 41 energy values uniformly distributed between $8.96 \mathrm{meV}$ and $9.04 \mathrm{meV}$. The simulations have been performed using an envelope-function model and a numerical approach based on recursive Green's function, written using a mixed representation: in the real space along the transport direction and in the space of the eigenmodes in the transverse direction $^{70,77}$. We have found that for more than 4 barriers the Fano factor is close to $1 / 3$. This confirms that also in semiconductors more traditional than graphene the tilting of the barriers and the mode-mixing it generates can give rise to a diffusive transport regime.

\section{CONCLUSION}

We have studied an armchair graphene ribbon with a series of randomly spaced tunnel barriers, observing the influence of the geometric details of the device on its conductance and shot noise behavior, which is dominated by Klein tunneling and resonant tunneling through the states quasi-localized in the barriers.

From the results of our simulations, we conclude that the effect of resonant tunneling strongly depends on the similarity between the barriers: in the case of identical barriers, this structure actually represents an energy filter for the impinging carriers. Instead, Klein tunneling gives rise to mode-filtering, since modes with a smaller incidence angle experience larger transmission. For a high number of barriers two different transport regimes are reached, depending on the exact width of the ribbon: in semiconducting ribbons transport is strongly localized, while in metallic ribbons the lowest mode passes unaltered through the device.

We have analyzed also the case of tilted barriers, where we have shown that transport can become diffusive. Furthermore, we have verified that this is true not only in graphene, but also in more traditional semiconductors.

As we have shown in our simulations, this multiplicity of behaviors has a strong impact on the shot noise suppression factor, which approaches the values $0,1 / 3$, or 1 when perfect transmission, diffusive dynamics, or strong localization are reached, respectively. Therefore, the analysis and measurement of shot noise turns out to be a very useful tool for the study of the transport regime taking place in the device.

Since we have found that these effects should persist also in 
the case of barriers with a smooth profile and in the presence of low levels of potential disorder, they should be detectable in actual measurements. Experiments performed on graphene ribbons biased by a series of unevenly spaced negatively biased top gates would be useful to validate our analysis and could represent an important step forward towards the application of these devices as energy and mode filters in graphenebased electronics.

\section{ACKNOWLEDGMENTS}

This work was partially supported by the Italian Ministry of Education and Research (MIUR) in the framework of the CrossLab project (Departments of Excellence).

${ }^{1}$ K. S. Novoselov, A. K. Geim, S. V. Morozov, D. Jiang, Y. Zhang, S. V. Dubonos, I. V. Grigorieva, and A. A. Firsov, "Electric Field Effect in Atomically Thin Carbon Films," Science 306, 666-669 (2004), DOI: 10.1126/science. 1102896

${ }^{2}$ K. S. Novoselov, A. K. Geim, S. V. Morozov, D. Jiang, M. I. Katsnelson, I. V. Grigorieva, S. V. Dubonos, and A. A. Firsov, "Two-dimensional gas of massless Dirac fermions in graphene," Nature 438, 197-200 (2005), DOI: 10.1038 /nature 04233

${ }^{3}$ A. K. Geim and K. S. Novoselov, "The rise of graphene," Nat. Mater. 6, 183-191 (2007), DOI: 10.1038/nmat1849

${ }^{4}$ K. S. Novoselov, V. I. Fal'ko, L. Colombo, P. R. Gellert, M. G. Schwab, and K. Kim, “A roadmap for graphene," Nature 490, 192-200 (2012), DOI: 10.1038/nature11458

${ }^{5}$ P. Avouris and C. Dimitrakopoulos, "Graphene: synthesis and applications," Mater. Today 15, 86-97 (2012), DOI: 10.1016/S13697021(12)70044-5

${ }^{6}$ L. E. F. Foa Torres, S. Roche, and J.-C. Charlier, Introduction to GrapheneBased Nanomaterials: From Electronic Structure to Quantum Transport (Cambridge University Press, Cambridge, UK, 2014) ISBN: 1107030838

${ }^{7}$ H. Zhu, Z. Xu, D. Xie, and Y. Fang, Graphene: Fabrication, Characterizations, Properties and Applications (Academic Press, London, UK, 2018) ISBN: 978-0-12-812651-6

${ }^{8}$ A. C. Ferrari, F. Bonaccorso, V. Fal'ko, K. S. Novoselov, S. Roche, P. Bøggild, S. Borini, F. H. L. Koppens, V. Palermo, N. Pugno, J. A. Garrido, R. Sordan, A. Bianco, L. Ballerini, M. Prato, E. Lidorikis, J. Kivioja, C. Marinelli, T. Ryhänen, A. Morpurgo, J. N. Coleman, V. Nicolosi, L. Colombo, A. Fert, M. Garcia-Hernandez, A. Bachtold, G. F. Schneider, F. Guinea, C. Dekker, M. Barbone, Z. Sun, C. Galiotis, A. N. Grigorenko, G. Konstantatos, A. Kis, M. Katsnelson, L. Vandersypen, A. Loiseau, V. Morandi, D. Neumaier, E. Treossi, V. Pellegrini, M. Polini, A. Tredicucci, G. M. Williams, B. H. Hong, J.-H. Ahn, J. M. Kim, H. Zirath, B. J. van Wees, H. van der Zant, L. Occhipinti, A. Di Matteo, I. A. Kinloch, T. Seyller, E. Quesnel, X. Feng, K. Teo, N. Rupesinghe, P. Hakonen, S. R. T. Neil, Q. Tannock, T. Löfwander, and J. Kinaret, "Science and technology roadmap for graphene, related two-dimensional crystals, and hybrid systems," Nanoscale 7, 4598-4810 (2015), DOI: 10.1039/c4nr01600a

${ }^{9}$ H. Raza, Graphene Nanoelectronics: Metrology, Synthesis, Properties and Applications (Springer Verlag, Heidelberg, Germany, 2012) ISBN: 9783642204678

${ }^{10}$ M. Poljak, E. B. Song, M. Wang, T. Suligoj, and K. L. Wang, "Influence of Edge Defects, Vacancies, and Potential Fluctuations on Transport Properties of Extremely Scaled Graphene Nanoribbons," IEEE Trans. Electron Devices 59, 3231-3238 (2012), DOI: 10.1109/TED.2012.2217969

${ }^{11}$ B. Pellegrini, P. Marconcini, M. Macucci, G. Fiori, and G. Basso, "Carrier density dependence of $1 / f$ noise in graphene explained as a result of the interplay between band-structure and inhomogeneities," J. Stat. Mech. Theor. Exp. 2016, 054017 (2016), DOI: 10.1088/1742-5468/2016/05/054017

${ }^{12} \mathrm{X}$. Li, L. Cao, H.-L. Li, H. Wan, and G. Zhou, "Spin-Resolved Transport Properties of a Pyridine-Linked Single Molecule Embedded between Zigzag-Edged Graphene Nanoribbon Electrodes," J. Phys. Chem. C 120, 3010-3018 (2016), DOI: 10.1021/acs.jpcc.5b10880
${ }^{13}$ X.-K. Chen, J. Liu, Z.-X. Xie, Y. Zhang, Y.-X. Deng, and K.-Q. Chen, “A local resonance mechanism for thermal rectification in pristine/branched graphene nanoribbon junctions," Appl. Phys. Lett. 113, 121906 (2018), DOI: $10.1063 / 1.5053233$

${ }^{14}$ A. H. Castro Neto, F. Guinea, N. M. R. Peres, K. S. Novoselov, and A. K. Geim, "The electronic properties of graphene," Rev. Mod. Phys. 81, 109162 (2009), DOI: 10.1103/RevModPhys.81.109

${ }^{15}$ T. Enoki and T. Ando, Physics and Chemistry of Graphene: Graphene to Nanographene (CRC Press, Boca Raton, FL, 2013) ISBN: 9789814241489

${ }^{16}$ R. Saito, G. Dresselhaus, and M. S. Dresselhaus, Physical Properties of Carbon Nanotubes (Imperial College Press, London, UK, 1998) ISBN: 1860940935

${ }^{17} \mathrm{P}$. Marconcini and M. Macucci, "The k·p method and its application to graphene, carbon nanotubes and graphene nanoribbons: the Dirac equation," Riv. Nuovo Cimento 34, 489-584 (2011), DOI: 10.1393/ncr/i201110068-1

${ }^{18}$ T. Ando, "Theory of Electronic States and Transport in Carbon Nanotubes," J. Phys. Soc. Japan 74, 777-817 (2005), DOI: 10.1143/JPSJ.74.777

${ }^{19}$ M. I. Katsnelson and K. S. Novoselov, "Graphene: New bridge between condensed matter physics and quantum electrodynamics," Solid State Commun. 143, 3-13 (2007), DOI: 10.1016/j.ssc.2007.02.043

${ }^{20}$ M. I. Katsnelson, "Graphene: carbon in two dimensions," Mater. Today 10, 20-27 (2007), DOI: 10.1016/S1369-7021(06)71788-6

${ }^{21}$ M. I. Katsnelson, K. S. Novoselov, and A. K. Geim, "Chiral tunnelling and the Klein paradox in graphene," Nat. Phys. 2, 620-625 (2006), DOI: $10.1038 /$ nphys 384

${ }^{22}$ T. Tudorovskiy, K. J. A. Reijnders, and M. I. Katsnelson, "Chiral tunneling in single-layer and bilayer graphene," Phys. Scr. T146, 014010 (2012), DOI: $10.1088 / 0031-8949 / 2012 / T 146 / 014010$

${ }^{23}$ C. W. J. Beenakker, "Colloquium: Andreev reflection and Klein tunneling in graphene," Rev. Mod. Phys. 80, 1337-1354 (2008), DOI: 10.1103/RevModPhys.80.1337

${ }^{24}$ A. F. Young and P. Kim, "Quantum interference and Klein tunnelling in graphene heterojunctions," Nat. Phys. 5, 222-226 (2009), DOI: $10.1038 /$ nphys 1198

${ }^{25}$ N. Stander, B. Huard, and D. Goldhaber-Gordon, "Evidence for Klein Tunneling in Graphene p-n Junctions,” Phys. Rev. Lett. 102, 026807 (2009), DOI: 10.1103/PhysRevLett.102.026807

${ }^{26}$ V. V. Cheianov and V. I. Fal'ko, "Selective transmission of Dirac electrons and ballistic magnetoresistance of n-p junctions in graphene," Phys. Rev. B 74, 041403(R) (2006), DOI: 10.1103/PhysRevB.74.041403

${ }^{27}$ J. Tworzydło, B. Trauzettel, M. Titov, A. Rycerz, and C. W. J. Beenakker, "Sub-Poissonian Shot Noise in Graphene," Phys. Rev. Lett. 96, 246802 (2006), DOI: 10.1103/PhysRevLett.96.246802

${ }^{28}$ Y. Zhang, Y.-W. Tan, H. L. Stormer, and P. Kim, "Experimental observation of the quantum Hall effect and Berry's phase in graphene," Nature 438, 201-204 (2005), DOI: 10.1038/nature04235

${ }^{29}$ K. S. Novoselov, E. McCann, S. V. Morozov, V. I. Fal'ko, M. I. Katsnelson, U. Zeitler, D. Jiang, F. Schedin, and A. K. Geim, "Unconventional quantum Hall effect and Berry's phase of $2 \pi$ in bilayer graphene," Nat. Phys. 2, 177 180 (2006), DOI: $10.1038 /$ nphys 245

${ }^{30}$ M. R. Connolly, R. K. Puddy, D. Logoteta, P. Marconcini, M. Roy, J. P. Griffiths, G. A. C. Jones, P. A. Maksym, M. Macucci, and C. G. Smith, "Unraveling Quantum Hall Breakdown in Bilayer Graphene with Scanning Gate Microscopy," Nano Lett. 12, 5448-5454 (2012), DOI: $10.1021 / \mathrm{nl} 3015395$

${ }^{31}$ V. V. Cheianov, V. Fal'ko, and B. L. Altshuler, "The Focusing of Electron Flow and a Veselago Lens in Graphene p-n Junctions," Science 315, 1252 1255 (2007), DOI: $10.1126 /$ science. 1138020

${ }^{32}$ G.-H. Lee, G.-H. Park, and H.-J. Lee, "Observation of negative refraction of Dirac fermions in graphene," Nat. Phys. 11, 925-929 (2015), DOI: $10.1038 /$ nphys 3460

${ }^{33}$ P. E. Allain and J. N. Fuchs, "Klein tunneling in graphene: optics with massless electrons," Eur. Phys. J. B 83, 301-317 (2011), DOI: 10.1140/epjb/e2011-20351-3

${ }^{34}$ V. N. Do, V. H. Nguyen, P. Dollfus, and A. Bournel, "Electronic transport and spin-polarization effects of relativisticlike particles in mesoscopic graphene structures," J. Appl. Phys. 104, 063708 (2008), DOI: $10.1063 / 1.2980045$

${ }^{35} \mathrm{~V}$. H. Nguyen, Electronic transport and spin polarization effects in 
graphene nanostructures, Ph.D. thesis, Paris-Sud University, U. F. R. Scientific Campus in Orsay, France (2010)

${ }^{36}$ E. Rossi, J. H. Bardarson, P. W. Brouwer, and S. Das Sarma, "Signatures of Klein tunneling in disordered graphene p-n-p junctions," Phys. Rev. B 81, 121408(R) (2010), DOI: 10.1103/PhysRevB.81.121408

${ }^{37}$ J. Velasco Jr., G. Liu, W. Bao, and C. N. Lau, "Electrical transport in highquality graphene pnp junctions," New J. Phys. 11, 095008 (2009), DOI: 10.1088/1367-2630/11/9/095008

${ }^{38}$ E. D. Herbschleb, R. K. Puddy, P. Marconcini, J. P. Griffiths, G. A. C. Jones, M. Macucci, C. G. Smith, and M. R. Connolly, "Direct imaging of coherent quantum transport in graphene p-n-p junctions," Phys. Rev. B 92, 125414 (2015), DOI: 10.1103/PhysRevB.92.125414

${ }^{39}$ L. Brey and H. A. Fertig, "Electronic states of graphene nanoribbons studied with the Dirac equation," Phys. Rev. B 73, 235411 (2006), DOI: 10.1103/PhysRevB.73.235411

${ }^{40}$ Y.-W. Son, M. L. Cohen, and S. G. Louie, "Energy gaps in graphene nanoribbons," Phys. Rev. Lett. 97, 216803 (2006), DOI: 10.1103/PhysRevLett.97.216803

${ }^{41} \mathrm{~K}$. Wakabayashi and T. Aoki, "Electrical Conductance of Zigzag Nanographite Ribbons with Locally Applied Gate Voltage," Int. J. Mod. Phys. B 16, 4897-4909 (2002), DOI: 10.1142/S0217979202014917

${ }^{42}$ J. Nakabayashi, D. Yamamoto, and S. Kurihara, "Band-Selective Filter in a Zigzag Graphene Nanoribbon," Phys. Rev. Lett. 102, 066803 (2009), DOI: 10.1103/PhysRevLett.102.066803

${ }^{43}$ A. R. Akhmerov, J. H. Bardarson, A. Rycerz, and C. W. J. Beenakker, "Theory of the valley-valve effect in graphene nanoribbons," Phys. Rev. B 77, 205416 (2008), DOI: 10.1103/PhysRevB.77.205416

${ }^{44}$ O. Roslyak, A. Iurov, G. Gumbs, and D. Huang, "Unimpeded tunneling in graphene nanoribbons," J. Phys. Condens. Matter 22, 165301 (2010), DOI: 10.1088/0953-8984/22/16/165301

${ }^{45}$ L. Brey and H. A. Fertig, "Emerging Zero Modes for Graphene in a Periodic Potential," Phys. Rev. Lett. 103, 046809 (2009), DOI: 10.1103/PhysRevLett.103.046809

${ }^{46}$ H. A. Fertig and L. Brey, "Mesoscopics in Graphene: Dirac Points in Periodic Geometries," in Graphene Nanoelectronics: Metrology, Synthesis, Properties and Applications, edited by H. Raza (Springer Verlag, Heidelberg, Germany, 2012) Chap. 10, pp. 301-323, DOI: 10.1007/978-3-64222984-8_10, ISBN: 9783642204678

${ }^{47}$ P. Burset, A. L. Yeyati, L. Brey, and H. A. Fertig, "Transport in superlattices on single-layer graphene," Phys. Rev. B 83, 195434 (2011), DOI: 10.1103/PhysRevB.83.195434

${ }^{48}$ C.-H. Park, Y.-W. Son, L. Yang, M. L. Cohen, and S. G. Louie, "Landau Levels and Quantum Hall Effect in Graphene Superlattices," Phys. Rev. Lett. 103, 046808 (2009), DOI: 10.1103/PhysRevLett.103.046808

${ }^{49}$ M. Barbier, P. Vasilopoulos, and F. M. Peeters, "Extra Dirac points in the energy spectrum for superlattices on single-layer graphene," Phys. Rev. B 81, 075438 (2010), DOI: 10.1103/PhysRevB.81.075438

${ }^{50}$ M. Barbier, P. Vasilopoulos, and F. M. Peeters, "Single-layer and bilayer graphene superlattices: collimation, additional Dirac points and Dirac lines," Philos. Trans. Royal Soc. A 368, 5499-5524 (2010), DOI: 10.1098/rsta.2010.0218

${ }^{51}$ S. Dubey, V. Singh, A. K. Bhat, P. Parikh, S. Grover, R. Sensarma, V. Tripathi, K. Sengupta, and M. M. Deshmukh, "Tunable Superlattice in Graphene To Control the Number of Dirac Points," Nano Lett. 13, 39903995 (2013), DOI: 10.1021/n14006029

${ }^{52}$ G. J. Xu, X. G. Xu, B. H. Wu, J. C. Cao, and C. Zhang, "The resonant tunneling through a graphene multiquantum well system," J. Appl. Phys. 107, 123718 (2010), DOI: 10.1063/1.3445782

${ }^{53}$ J. M. Pereira Jr., F. M. Peeters, A. Chaves, and G. A. Farias, "Klein tunneling in single and multiple barriers in graphene," Semicond. Sci. Technol. 25, 033002 (2010), DOI: 10.1088/0268-1242/25/3/033002

${ }^{54} \mathrm{Y} . \mathrm{X}$. Li and L.-F. Xu, "Shot noise suppression in a series graphene tunnel barrier structure," Solid State Commun. 151, 219-222 (2011), DOI: $10.1016 /$ j.ssc.2010.11.029

${ }^{55}$ W. Schottky, "Über spontane Stromschwankungen in verschiedenen Elektrizitätsleitern,” Ann. Phys. 362, 541-567 (1918), DOI: 10.1002/andp. 19183622304

${ }^{56}$ J. H. Oh and D. Ahn, "Klein Tunnelling Through an Oblique Barrier in Graphene Ribbons," J. Korean Phy. Soc. 59, 2786-2791 (2011), DOI:
$10.3938 / \mathrm{jkps} .59 .2786$

${ }^{57}$ P. Marconcini, M. Macucci, G. Iannaccone, and B. Pellegrini, "Quantum analysis of shot noise suppression in a series of tunnel barriers," Phys. Rev. B 79, 241307(R) (2009), DOI: 10.1103/PhysRevB.79.241307

${ }^{58} \mathrm{P}$. Marconcini, M. Macucci, D. Logoteta, and M. Totaro, "Is the regime with shot noise suppression by a factor $1 / 3$ achievable in semiconductor devices with mesoscopic dimensions?" Fluct. Noise Lett. 11, 1240012 (2012), DOI: $10.1142 / \mathrm{S} 0219477512400123$

${ }^{59}$ D. Logoteta, P. Marconcini, C. Bonati, M. Fagotti, and M. Macucci, "High-performance solution of the transport problem in a graphene armchair structure with a generic potential," Phys. Rev. E 89, 063309 (2014), DOI: 10.1103/PhysRevE.89.063309

${ }^{60} \mathrm{P}$. Marconcini and M. Macucci, "Symmetry-dependent transport behavior of graphene double dots," J. Appl. Phys. 114, 163708 (2013), DOI: $10.1063 / 1.4827382$

${ }^{61}$ P. Marconcini and M. Macucci, "Envelope-Function-Based Transport Simulation of a Graphene Ribbon With an Antidot Lattice," IEEE Trans. Nanotechnol. 16, 534-544 (2017), DOI: 10.1109/TNANO.2016.2645663

${ }^{62}$ M. Fagotti, C. Bonati, D. Logoteta, P. Marconcini, and M. Macucci, "Armchair graphene nanoribbons: $\mathscr{P} \mathscr{T}$-symmetry breaking and exceptional points without dissipation," Phys. Rev. B 83, 241406(R) (2011), DOI: 10.1103/PhysRevB.83.241406

${ }^{63}$ P. Marconcini, D. Logoteta, and M. Macucci, "Sinc-based method for an efficient solution in the direct space of quantum wave equations with periodic boundary conditions," J. Appl. Phys. 114, 173707 (2013), DOI: $10.1063 / 1.4828875$

${ }^{64}$ S. Datta, Electronic Transport in Mesoscopic Systems (Cambridge University Press, Cambridge, UK, 1997) ISBN: 9780521599436

${ }^{65} \mathrm{R}$. Landauer, "Spatial variation of currents and fields due to localized scatterers in metallic conduction," IBM J. Res. Dev. 1, 223-231 (1957), DOI: $10.1147 /$ rd.13.0223

${ }^{66} \mathrm{M}$. Büttiker, Y. Imry, R. Landauer, and S. Pinhas, "Generalized manychannel conductance formula with application to small rings," Phys. Rev. B 31, 6207-6215 (1985), DOI: 10.1103/PhysRevB.31.6207

${ }^{67} \mathrm{M}$. Büttiker, "Scattering theory of thermal and excess noise in open conductors," Phys. Rev. Lett. 65, 2901-2904 (1990), DOI: 10.1103/PhysRevLett.65.2901

${ }^{68} \mathrm{M}$. Macucci and P. Marconcini, "Shot noise suppression due to a magnetic field in disordered conductors," J. Comput. Electron. 14, 107-113 (2015), DOI: $10.1007 / \mathrm{s} 10825-014-0647-\mathrm{Z}$

${ }^{69}$ E. Anderson, Z. Bai, C. Bischof, S. Blackford, J. Demmel, J. Dongarra, J. Du Croz, A. Greenbaum, S. Hammarling, A. McKenney, and D. Sorensen, LAPACK Users' Guide (Society for Industrial and Applied Mathematics, Philadelphia, PA, 1999) ISBN: 0898714478

${ }^{70}$ F. Sols, M. Macucci, U. Ravaioli, and K. Hess, "Theory for a quantum modulated transistor," J. Appl. Phys. 66, 3892-3906 (1989), DOI: $10.1063 / 1.344032$

${ }^{71}$ F. Sols, M. Macucci, U. Ravaioli, and K. Hess, "On the possibility of transistor action based on quantum interference phenomena," Appl. Phys. Lett. 54, 350-352 (1989), DOI: 10.1063/1.100966

${ }^{72}$ L. Esaki, "Long journey into tunneling," Rev. Mod. Phys. 46, 237-244 (1974), DOI: 10.1103/RevModPhys.46.237

${ }^{73}$ X. Pan, W. Qiu, and E. Skafidas, "Energy-filtered Electron Transport Structures for Low-power Low-noise 2-D Electronics," Sci. Rep. 6, 36167 (2016), DOI: $10.1038 /$ srep36167

${ }^{74}$ A. Kumar, L. Saminadayar, D. C. Glattli, Y. Jin, and B. Etienne, "Experimental Test of the Quantum Shot Noise Reduction Theory," Phys. Rev. Lett. 76, 2778-2781 (1996), DOI: 10.1103/PhysRevLett.76.2778

${ }^{75}$ N. T. T. Nguyen, D. Q. To, and V. L. Nguyen, "A model for ballistic transport across locally gated graphene bipolar junctions," J. Phys.: Condens. Matter 26, 015301 (2014), DOI: 10.1088/0953-8984/26/1/015301

${ }^{76} \mathrm{P}$. Marconcini and M. Macucci, "Approximate calculation of the potential profile in a graphene-based device," IET Circ. Device. Syst. 9, 30-38 (2015), DOI: 10.1049/iet-cds.2014.0003

${ }^{77}$ M. Macucci, A. Galick, and U. Ravaioli, "Quasi-three-dimensional Green's-function simulation of coupled electron waveguides," Phys. Rev. B 52, 5210-5220 (1995), DOI: 10.1103/PhysRevB.52.5210 